\title{
MULTILEVEL CONTROL VARIATES FOR UNCERTAINTY QUANTIFICATION IN SIMULATIONS OF CLOUD CAVITATION*
}

\author{
JONAS ŠUKYS ${ }^{\dagger}$, URSULA RASTHOFER ${ }^{\ddagger}$, FABIAN WERMELINGER ${ }^{\ddagger}$, PANAGIOTIS \\ HADJIDOUKAS ${ }^{\S}$, AND PETROS KOUMOUTSAKOS ${ }^{\natural}$
}

\begin{abstract}
We quantify uncertainties in the location and magnitude of extreme pressure spots revealed from large scale multiphase flow simulations of cloud cavitation collapse. We examine clouds containing 500 cavities and quantify uncertainties related to their initial spatial arrangement. The resulting 2,000-dimensional space is sampled using a nonintrusive and computationally efficient multilevel Monte Carlo (MLMC) methodology. We introduce novel empirically optimal control variate coefficients to enhance the variance reduction in MLMC. The proposed multilevel control variates Monte Carlo leads to more than two orders of magnitude speedup when compared to standard Monte Carlo methods. We identify large uncertainties in the location and magnitude of the peak pressure pulse and present its statistical correlations and joint probability density functions with the geometrical characteristics of the cloud. Characteristic properties of spatial cloud structure are identified as potential causes of significant uncertainties in exerted collapse pressures.
\end{abstract}

Key words. compressible multiphase flow, high performance computing, diffuse interface method, bubble collapse, cloud cavitation, uncertainty quantification, multilevel Monte Carlo, control variates, fault tolerance

AMS subject classifications. 76B10, 68W10, 65C05, 65C60, 68M15

DOI. $10.1137 / 17 \mathrm{M} 1129684$

1. Introduction. Cloud cavitation collapse pertains to the inception of multiple gas cavities in a liquid, and their subsequent rapid collapse driven by an increase of the ambient pressure. Shock waves emanate from the cavities with pressure peaks up to two orders of magnitude larger than the ambient pressure $[50,13,8]$. When such shock waves impact on solid walls, they may cause material erosion, considerably reducing the performance and longevity of turbomachinery and fuel injection engines $[69,41]$. On the other hand, the destructive potential of cavitation can be harnessed for noninvasive biomedical applications $[18,9]$ and efficient travel in military sub-surface applications [6]. Prevalent configurations of cavitation often involve

*Submitted to the journal's Computational Methods in Science and Engineering section May 10, 2017; accepted for publication (in revised form) August 6, 2018; published electronically October 16,
2018 .

http://www.siam.org/journals/sisc/40-5/M112968.html

Funding: Authors acknowledge support from the following organizations: Innovative and Novel Computational Impact on Theory and Experiment (INCITE) program, for awarding computer time under the project CloudPredict; Argonne Leadership Computing Facility, which is a DOE Office of Science User Facility supported under Contract DE-AC02-06CH11357, for providing access and support for MIRA, CETUS, and COOLEY systems. Partnership for Advanced Computing in Europe (PRACE) projects PRA091 and Pra09_2376, together with Jülich and CINECA Supercomputing Centers; Swiss National Supercomputing Center (CSCS) for computational resources grant under project ID s500; European Research Council (ERC) Advanced Investigator Award 341117 (FMCoBe).

'Computational Science and Engineering Laboratory, ETH Zürich, Switzerland. Current address: Eawag, Swiss Federal Institute of Aquatic Science and Technology, Switzerland (jonas.sukys@ eawag.ch, http://science.sukys.ch).

Computational Science and Engineering Laboratory, ETH Zürich, Switzerland (urasthofer@ethz. ch, fabianw@mavt.ethz.ch).

${ }_{\S}$ Computational Science and Engineering Laboratory, ETH Zürich, Switzerland. Current address: IBM Research-Zurich, Switzerland (hat@zurich.ibm.com).

" Corresponding author. Computational Science and Engineering Laboratory, ETH Zürich, Switzerland (petros@mavt.ethz.ch).

$$
\mathrm{B} 1361
$$

Copyright (C) by SIAM. Unauthorized reproduction of this article is prohibited. 

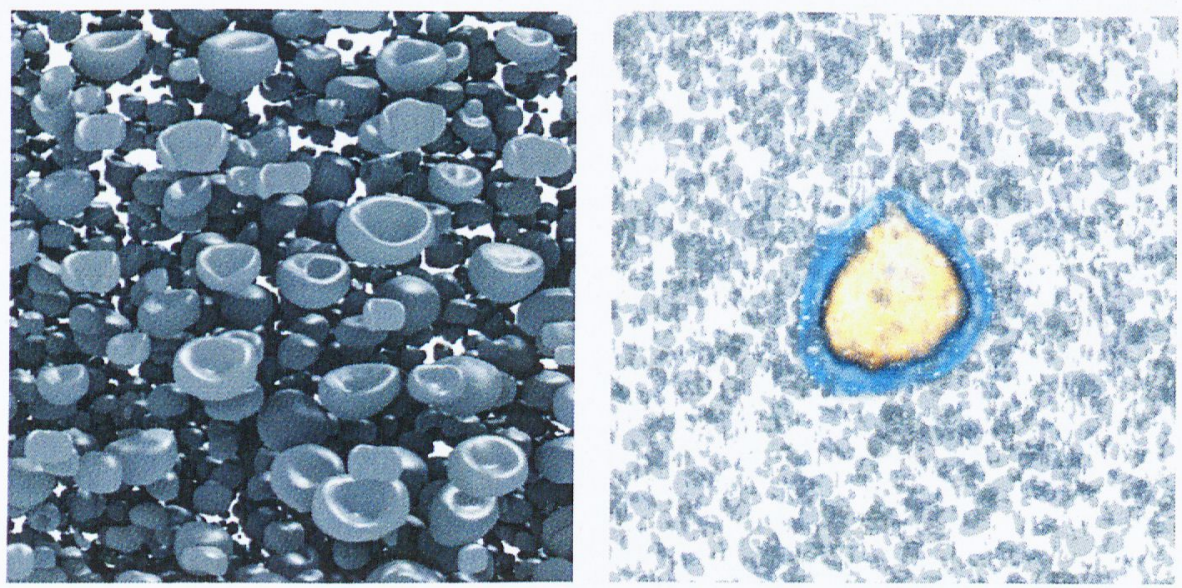

FIG. 1. Surface view of a collapsing cloud containing 50,000 gas cavities (left) and pressure peak at the center of the cloud (right). Outer bubbles evolve into caplike shapes, forming an array of inwards directed velocity microjets. The resulting focused pressure peak amplifications of two orders of magnitude are exerted at the cloud center [31].

clouds of hundreds or thousands of bubbles [8]. The cloud collapse entails a location dependent, often nonspherical, collapse of each cavity that progresses from the surface to the center of the cloud. Pressure waves emanating from collapsing cavities located near the surface of the cloud act as amplifiers to the subsequent collapses at the center of the cloud. The interaction of these pressure waves increases the destructive potential as compared to the single bubble case. Cavitation, in particular, as it occurs in realistic conditions, presents a formidable challenge to experimental $[7,11,84]$ and computational $[1,77]$ studies due to its geometric complexity and multitude of spatiotemporal scales. Blake et al. [5] studied the single bubble asymmetric collapse using a boundary integral method. Johnsen and Colonius [37] investigated the potential damage of single collapsing bubbles in both spherical and asymmetric regimes for a range of pulse peak pressures in shock-induced collapse. Lauer et al. [40] studied collapses of arrays of cavities under shock waves using the sharp interface technique of $\mathrm{Hu}$ et al. [34]. Recent numerical investigation of cloud cavitation involved a cluster of 125 vapor bubbles inside a pressurized liquid at 40 bar $[19,1]$, and a cluster of 2,500 gas bubbles with ambient liquid pressure of 100 bar [66]. Large scale numerical simulations of cloud cavitation collapse considered clouds containing 50,000 bubbles [31]. Visualizations of such a collapsing cloud and the resulting focused pressure peak at the center are reproduced in Figure 1. However, the computational demands of these simulations do not allow for further parametric studies.

A challenge in modeling and quantifying cloud cavitation collapse is the dependence of critical quantities of interest (QoIs), such as peak pressure or collapse time, on a particular (random) cloud configuration [77] (see also Figure 2). The systematic study of such dependencies can be addressed through an uncertainty quantification (UQ) framework, recently applied in $[17,6]$. In $[46,47,48]$, a mathematical framework was provided for uncertain solutions of hyperbolic equations. Popular probability space discretization methods include generalized polynomial chaos techniques (see $[14,42,79,63,80,27]$ and references therein). An alternative class of methods for quantifying uncertainty in PDEs are the stochastic collocation methods $[83,44,82]$.

Copyright (C) by SIAM. Unauthorized reproduction of this article is prohibited. 


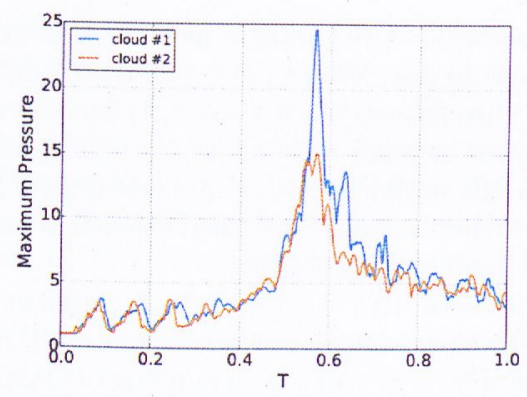

FIG. 2. Evolution of the normalized peak pressure versus normalized time in free-field collapse of two spherical clouds containing 1,000 bubbles. Two samples are drawn from the same uniform distribution (for the positions of the cavities) and log-normal distribution (for the radii of the cavities).

However, the lack of regularity of the solution with respect to the stochastic variables impedes efficient performance of both the stochastic Galerkin as well as the stochastic collocation methods, in particular, for high-dimensional parameter spaces. Here, we propose the development and implementation of nonintrusive Monte Carlo (MC) methods for UQ of cloud cavitation collapse. In MC methods, the governing equations are solved for a sequence of randomly generated samples, which are combined to ascertain statistical information. However, the robustness of MC methods with respect to solution regularity comes at the price of a low error convergence rate regarding the number of samples. Drawbacks of the aforementioned numerical UQ methods inspired the development of various multifidelity methods, such as Multi-Level MC (MLMC) [24], Further developments include multifidelity Gaussian process regression based on cokriging [56], and purely data-driven algorithms for linear equations using Gaussian process priors to completely circumvent the use of numerical discretization schemes [65]. MLMC methods were introduced by Heinrich for numerical quadrature [32], then pioneered by Giles for Itô SPDEs [24], and have been lately applied to various stochastic PDEs $[3,15,26,53]$. The MLMC algorithm was also extended to hyperbolic conservation laws and to massively parallel simulations of the random multidimensional Euler, magnetohydrodynamics, and shallow water equations $[46,47,48,49,75,72,43]$. Subsequent MLMC improvements include Bayesian inference for fusing knowledge on empirical statistical estimates and deterministic convergence rates [16], an alternative multifidelity setting for nonhierarchical structure of the resolution levels with jointly optimized control variate coefficients and the number of required MC samples (with a posteriori rounding to integers) $[60,61]$, multilevel control variates with a low-rank approximation to the solutions on coarser grids [22], and multilevel Markov chain MC posterior sampling in Bayesian inference problems [20, 21].

The ensemble uncertainty of the clouds is parametrized by means of probability distributions of cavity radii, positions, and initial internal pressures. Our goal is to perform simulations of cloud cavitation collapse with an unprecedented number of interacting cavities with full UQ on QoIs (peak pressure magnitudes, locations, and cloud collapse times) in terms of means, variances, confidence intervals, and even probability density functions. To the best of our knowledge, such extensive UQ analysis of (even small) clouds has not been accomplished before. We propose an improved non-intrusive MLMC method with novel empirically optimal control variate coefficients, termed MLCV. Design desicions in MLCV partially overlap with some of the MLMC improvements mentioned in the previous paragragh, with a specific focus of 
MLCV being to achieve efficient UQ for black-box type deterministic models on large high performance computing (HPC) systems. We do our best to point out these similarities as well as highlight various differences throughout the manuscript; however, we acknowledge that a more elaborate comparison of these methodologies is beyond the scope of our work. MLCV is coupled with the state-of-the-art numerical solver for cloud cavitation collapse simulations and provides robust statistical estimates on relevant QoIs, emphasizing the importance of UQ in such problems.

The paper is structured as follows: section 2 introduces the governing multiphase equations and the finite volume method used for their solution. It also presents the empirically optimal control variate MLMC method for the statistical sampling of QoIs. Numerical experiments quantifying such uncertainties and identifying their relations to the geometric properties of the cloud by means of joint probability density function estimates are reported in section 3. We summarize our findings in section 4.

2. Computational methods. The governing system of equations for multiphase flows are discretized using a finite volume method (FVM) that is efficiently implemented so as to take advantage of supercomputer architectures. The sampling needed to estimate statistical properties from an ensemble of evolved QoIs is performed by a novel MLCV method. We introduce a Python implementation PyMLMC [74] of MLCV and its embedding in an open source UQ framework [29].

2.1. Governing equations. The dynamics of cavitating and collapsing clouds of bubbles are governed by the compressibility of the flow, with viscous dissipation and capillary effects taking place at orders of magnitude slower time scales. Hence, we describe cloud cavitation collapse through the Euler equations for inviscid, compressible, multiphase flows. The system of equations, derived from the Baer-Nunziato model [2], describes the evolution of density, momentum, and total energy of the multiphase flow in the domain $D \subset \mathbb{R}^{3}$ as in $[54,62]$ :

$$
\begin{aligned}
\frac{\partial \alpha_{1} \rho_{1}}{\partial t}+\nabla \cdot\left(\alpha_{1} \rho_{1} \mathbf{u}\right) & =0, \\
\frac{\partial \alpha_{2} \rho_{2}}{\partial t}+\nabla \cdot\left(\alpha_{2} \rho_{2} \mathbf{u}\right) & =0, \\
\frac{\partial(\rho \mathbf{u})}{\partial t}+\nabla \cdot(\rho \mathbf{u} \otimes \mathbf{u}+p \mathbf{I}) & =\mathbf{0}, \\
\frac{\partial E}{\partial t}+\nabla \cdot((E+p) \mathbf{u}) & =0, \\
\frac{\partial \alpha_{2}}{\partial t}+\mathbf{u} \cdot \nabla \alpha_{2} & =K \nabla \cdot \mathbf{u},
\end{aligned}
$$

where

$$
K=\frac{\alpha_{1} \alpha_{2}\left(\rho_{1} c_{1}^{2}-\rho_{2} c_{2}^{2}\right)}{\alpha_{1} \rho_{2} c_{2}^{2}+\alpha_{2} \rho_{1} c_{1}^{2}} .
$$

All quantities, unless otherwise stated, depend on spatial variable $\mathrm{x} \in D$ and time variable $t \geq 0$. This system comprises two mass conservation equations, one for each phase, conservation equations for momentum and total energy in single- or mixture-fluid formulation, as well as a transport equation for the volume fraction of one of the two phases with the source/sink term on the right-hand side. In (1)-(5), $\mathrm{u}$ denotes the velocity, $p$ the pressure, I the identity tensor, $\rho$ the (mixture) density, $E$ the (mixture) total energy $E=\rho e+1 / 2 \rho(\mathbf{u} \cdot \mathbf{u})$, where $e$ is the (mixture) specific 
internal energy. Moreover, $\rho_{k}, \alpha_{k}$, and $c_{k}$ with $k \in\{1,2\}$ are density, volume fraction, and speed of sound of the two phases. For the mixture quantities, the following additional relations hold: $\alpha_{1}+\alpha_{2}=1, \rho=\alpha_{1} \rho_{1}+\alpha_{2} \rho_{2}$, and $\rho e=\alpha_{1} \rho_{1} e_{1}+\alpha_{2} \rho_{2} e_{2}$. We do not account for mass transfer between different phases (evaporation or condensation), so that the above equations describe a multicomponent flow. The source term in (5) for homogeneous mixtures [38] describes the reduction of the gas volume fraction in a mixture of gas and liquid when a compression wave travels across the mixing region and vice versa for an expansion wave. For a more detailed analysis on the positive influence of this term on the accuracy of the model equations, we refer to [66].

The equation system is closed by an appropriate equation of state for each of the phases. We employ the stiffened equation of state (see [45] for a review) to capture liquids and gases. This enables a simple, analytic approximation to arbitrary fluids and is expressed by

$$
p=\left(\gamma_{k}-1\right) \rho_{k} e_{k}-\gamma_{k} p_{\mathrm{c}, k},
$$

where isobaric closure is assumed [62]. Parameters $\gamma_{k}$ and $p_{\mathrm{c}, k}$ depend on the material. For $p_{c, k}=0$, the equation of state for ideal gases is recovered. For the simulations in this manuscript, $\gamma_{1}=6.59$ and $p_{\mathrm{c}, 1}=4.049 \cdot 10^{8} \mathrm{~Pa}$ are used for water and $\gamma_{2}=1.4$ and $p_{\mathrm{c}, 2}=0 \mathrm{~Pa}$ for air.

2.2. Numerical method. The governing system $(1)-(5)$ can be recast into the
si-conservative form quasi-conservative form

$$
\frac{\partial \mathbf{Q}}{\partial t}+\frac{\partial \mathbf{F}(\mathbf{Q})}{\partial x}+\frac{\partial \mathbf{G}(\mathbf{Q})}{\partial y}+\frac{\partial \mathbf{H}(\mathbf{Q})}{\partial z}=\mathbf{R}(\mathbf{Q})
$$

where $\mathbf{Q}=\left(\alpha_{1} \rho_{1}, \alpha_{2} \rho_{2}, \rho \mathbf{u}, E, \alpha_{2}\right)^{T}$ is the vector of conserved (except for $\alpha_{2}$ which has a nonzero source term) variables and $F(Q), G(Q), H(Q)$ are vectors of flux functions

$$
\mathbf{F}(\mathbf{Q})=\left(\begin{array}{c}
\alpha_{1} \rho_{1} u_{x} \\
\alpha_{2} \rho_{2} u_{x} \\
\rho u_{x}^{2}+p \\
\rho u_{y} u_{x} \\
\rho u_{z} u_{x} \\
(E+p) u_{x} \\
\alpha_{2} u_{x}
\end{array}\right), \quad \mathbf{G}(\mathbf{Q})=\left(\begin{array}{c}
\alpha_{1} \rho_{1} u_{y} \\
\alpha_{2} \rho_{2} u_{y} \\
\rho u_{x} u_{y} \\
\rho u_{y}^{2}+p \\
\rho u_{z} u_{y} \\
(E+p) u_{y} \\
\alpha_{2} u_{y}
\end{array}\right), \quad \mathbf{H}(\mathbf{Q})=\left(\begin{array}{c}
\alpha_{1} \rho_{1} u_{z} \\
\alpha_{2} \rho_{2} u_{z} \\
\rho u_{x} u_{z} \\
\rho u_{y} u_{z} \\
\rho u_{z}^{2}+p \\
(E+p) u_{z} \\
\alpha_{2} u_{z}
\end{array}\right)
$$

The source term $R(Q)$ has all elements equal to zero except the last one,

$$
\mathbf{R}(\mathbf{Q})_{7}=\alpha_{2}(\nabla \cdot \mathbf{u})+K \nabla \cdot \mathbf{u}
$$

which appears due to rewriting (5) in conservative form [36] and incorporating the present source term. For the system (8), initial condition $\mathrm{Q}(\mathrm{x}, t=0)=\mathrm{Q}_{0}(\mathrm{x})$ over the entire domain $x \in D$ as well as boundary conditions at $x \in \partial D$ for all times $t \geq 0$ need to be provided to complete the full specification of the multiphase flow problem. The method of lines is applied to obtain a semidiscrete representation of (8), where space continuous operators are approximated using the FVM for uniform structured grids. The approach yields a system of ordinary differential equations

$$
\frac{d \mathbf{V}(t)}{d t}=\mathcal{L}(\mathbf{V}(t))
$$

where $\mathbf{V}$ is a vector of cell average values and $\mathcal{L}(\cdot)$ is a discrete operator that approx-

Copyright (C) by SIAM. Unauthorized reproduction of this article is prohibited. 
imates the convective fluxes and the given sources in the governing system. The temporal discretization of (9) is obtained by an explicit third-order low-storage RungeKutta scheme [81]. The computation of the numerical fluxes is based on a Godunovtype scheme using the approximate HLLC Riemann solver originally introduced for single-phase flow problems in [78]. The Riemann initial states are determined by a shock capturing third- or fifth-order accurate WENO reconstruction (see [35]). Following [36], the reconstruction is carried out using primitive variables, and the HLLC Riemann solver is adapted to (5) to prevent oscillations at interface. The solution is advanced with a time step size that satisfies the Courant-Friedrichs-Lewy (CFL) condition. For the coefficient weights in the Runge-Kutta stages, the values suggested in [28] are used, resulting in a total variation diminishing scheme.

2.3. Cubism-MPCF. The FVM used for the spatiotemporal numerical discretization of the nonlinear system of conservation laws in (8) is implemented in the open source software Cubism-MPCF $[68,30,31,66]$. The applied scheme entails three computational kernels: computation of CFL-limited time-step size $\Delta t$ based on a global reduction, evaluation of the approximate Riemann problem corresponding to the evaluation of the right-hand side $\mathcal{L}$ in $(9)$ for each time step, and appropriate Runge-Kutta update steps. The solver is parallelized with a hybrid paradigm using the MPI and OpenMP programming models. The software is split into three abstraction layers: cluster, node, and core [30]. The realization of the Cartesian domain decomposition and the interrank MPI communication is accomplished on the cluster layer. On the node layer, the thread level parallelism exploits the OpenMP standard using dynamic work scheduling. Spatial blocking techniques are used to increase locality of the data, with intrarank ghost cells obtained from loading fractions of the surrounding blocks, and interrank ghost cells obtained from a global buffer provided by the cluster layer. On the core layer, the actual computational kernels are executed, exploiting data level parallelism and instruction level parallelism, which are enabled by the conversion from an array-of-structures to a structure-of-arrays layout. For the simulations reported here, the main parts of the computations were executed in mixed precision arithmetic. More details on software design regarding the parallelization and optimization strategies used in Cubism-MPCF can be found in $[30,31,68,66]$. Cubism-MPCF has demonstrated state-of-the-art performance in terms of floating point operations, memory traffic, and storage, exhibiting almost perfect overlap of communication and computation [30,66]. The software has been optimized to take advantage of the IBM BlueGene/Q (BGQ) and Cray XC30 platforms to simulate cavitation collapse dynamics using up to 13 trillion computational cells with very efficient strong and weak scaling up to the full size of the MIRA (Argonne National Laboratory) and Piz Daint (Swiss Supercomputing Center) supercomputers $[68,31,66]$.

2.4. MLMC method. In this section, we introduce the MLMC framework for UQ. We also present a novel and improved numerical sampling method for approximating the statistical QoI.

This study is grounded on the theoretical probabilistic framework for nonlinear systems of conservation laws introduced in [47, 73]. Uncertainties in the system of conservation laws (8), such as uncertain initial data at time $t=0$ for the vector of conserved quantities $\mathrm{Q}$, are modeled as random fields [47, 73]. They depend on the spatial and temporal variables $\mathrm{x}$ and $t$, as well as on the stochastic parameter $\omega \in \Omega$, which represents variability in the cloud configuration. For instance, for uncertain 
initial data, we assume

$$
\mathrm{Q}_{0}(\mathrm{x}, \omega)=\mathrm{Q}(\mathrm{x}, 0, \omega) \in \mathbb{R}^{7}, \quad \mathrm{x} \in D, \quad \omega \in \Omega,
$$

i.e., at every spatial coordinate $\mathrm{x}$, initial data $\mathrm{Q}_{0}(\mathrm{x}, \omega)$ are a random vector containing 7 values, one for each equation in (8). We further assume that $Q_{0}$ is such that at every spatial point $\mathrm{x}$ the statistical moments such as expectation and variance exist and are defined by

$$
\mathbb{E}\left[\mathrm{Q}_{0}\right](\mathrm{x})=\int_{\Omega} \mathrm{Q}_{0}(\mathrm{x}, \omega) d \omega
$$

and

$$
\mathbb{V}\left[\mathrm{Q}_{0}\right](\mathrm{x})=\int_{\Omega}\left(\mathrm{Q}_{0}(\mathrm{x}, \omega)-\mathbb{E}\left[\mathrm{Q}_{0}\right](\mathrm{x})\right)^{2} d \omega .
$$

Such uncertainties, for instance in initial data $Q_{0}$, are propagated according to the dynamics governed by (8). Hence, the resulting evolved solution $\mathbf{Q}(\mathbf{x}, t, \omega)$ for $t>0$ is also a random field, called the random entropy solution; see $[47,73,46]$ for precise formulation and details.

2.4.1. The classical MLMC. The statistical moments of the QoIs, such as expectation $\mathbb{E}[q]$, are obtained through sampling by the MLMC methodology. Multilevel methods employ a hierarchy of spatial discretizations of the computational domain $D$ or, equivalently, a hierarchy of numerical deterministic solvers as described in subsection 2.2 , ordered by increasing "level of accuracy" $\ell=0, \ldots, L$. In many applications, which is especially relevant for On each such discretization for flow problems in multidimensional spatial domains. $\omega \in \Omega$, a numerical approximation for a given statistical realization (a "sample") denoted by $q_{\ell}(\omega)$.

The classical MLMC estimator [24] aims at providing accurate and computationally efficient estimates for statistical moments $[47,4]$ of $q$ in terms of the telescoping sum of numerical approximations $q_{\ell}(\omega)$ over all levels. In particular, an approximation $E^{\mathrm{MLMC}}\left[q_{L}\right]$ of the expected value $\mathbb{E}[q]$ is constructed from the approximate telescoping sum (neglecting the bias $\mathbb{E}\left[q-q_{L}\right]$ )

$$
\mathbb{E}[q] \approx \mathbb{E}\left[q_{L}\right]=\mathbb{E}\left[q_{0}\right]+\sum_{\ell=1}^{L}\left(\mathbb{E}\left[q_{\ell}\right]-\mathbb{E}\left[q_{\ell-1}\right]\right)
$$

by additionally approximating all exact expectations using Monte Carlo sampling with level-dependent number $M_{\ell}$ of samples for QoIs $q_{\ell}^{i}$, leading to

$$
\mathbb{E}\left[q_{L}\right] \approx E^{\mathrm{MLMC}}\left[q_{L}\right]=\frac{1}{M_{0}} \sum_{i=1}^{M_{0}} q_{0}^{i}+\sum_{\ell=1}^{L} \frac{1}{M_{\ell}} \sum_{i=1}^{M_{\ell}}\left(q_{\ell}^{i}-q_{\ell-1}^{i}\right) .
$$

We note that each sample $q_{\ell}^{i}$ is obtained by solving the governing system (8) using the FVM method from subsection 2.2 with discretization (number of mesh cells and time steps) corresponding to level $\ell$. Assuming that samples for $q_{0}^{i}$ and differences $q_{\ell}^{i}-q_{\ell-1}^{i}$ are drawn independently (however, sample pairs $q_{\ell}^{i}$ and $q_{\ell-1}^{i}$ are statistically dependent), the statistical mean square error of the (neglecting error due to the bias

Copyright (C) by SIAM. Unauthorized reproduction of this article is prohibited. 
term $\mathbb{E}\left[q-q_{L}\right]$, estimates of which for nonlinear hyperbolic systems are available only in special cases; see [47]) standard MLMC estimator is given by the sum of samplereduced variances of differences between every two consecutive levels,

$$
\varepsilon^{2}=\mathbb{E}\left[\left(E^{\mathrm{MLMC}}\left[q_{L}\right]-\mathbb{E}\left[q_{L}\right]\right)^{2}\right]=\frac{\mathbb{V}\left[q_{0}\right]}{M_{0}}+\sum_{\ell=1}^{L} \frac{\mathbb{V}\left[q_{\ell}-q_{\ell-1}\right]}{M_{\ell}} .
$$

An intuitive insight into the workings of the key MLMC advantage over standard MC is obtained by assuming that $\mathbb{V}\left[q_{\ell}\right] \approx \mathbb{V}[q]$ and observing that the MLMC sampling error in (15) can be approximated in terms of correlation coefficients of every two consecutive levels, i.e.,

$$
\varepsilon^{2} \approx \mathbb{V}[q]\left(\frac{1}{M_{0}}+2 \sum_{\ell=1}^{L} \frac{1-\operatorname{Cor}\left[q_{\ell}, q_{\ell-1}\right]}{M_{\ell}}\right) .
$$

Note, that strongly correlated QoIs on two consecutive levels lead to a significant reduction in the required number of samples on levels $\ell>0$. The optimal number of samples $M_{\ell}$ for each level $\ell=0, \ldots, L$ can be obtained using empirical or approximate asymptotic estimates on $\mathbb{V}\left[q_{0}\right]$ and $\mathbb{V}\left[q_{\ell}-q_{\ell-1}\right]$ by minimizing the amount of total computational work $M_{0} \mathbb{W}_{0}+\cdots+M_{L} \mathbb{W}_{L}$ for a prescribed error tolerance $\tau$ such that $\varepsilon \leq \tau$ in (15), as described in [24], or in order to avoid empirical rounding, as suggested in [57]; see also the forthcoming subsection 2.5 for additional remarks. Here, $\mathbb{W}_{0}$ denotes the amount of computational work needed to compute a single sample (statistical realization) on a given resolution level 0 . For levels $\ell>0$, $\mathbb{W}_{\ell}$ denotes the amount of computational work needed to compute a pair of such samples on resolution levels $\ell$ and $\ell-1$. The number of samples $M_{\ell}$ was shown to decrease exponentially with increasing level $\ell$, and hence such reduction directly translates into large computational savings over single-level $\mathrm{MC}$ sampling methods, as reported in $[46,47,48,75,72]$.

The proceeding generalization of a classical MLMC method builds upon an observation that the telescoping sum in (13) is not unique, i.e., there are many different ways to write such a telescoping sum involving approximations $q_{\ell}$ of $\mathrm{QoI} q$ and coarser resolutions levels $\ell$. In particular, some applications of a well-established control variate theory has been already explored in recent works, including $[55,59,61]$. We present a novel method for reducing the variance using control variates and further increasing the efficiency of the classical MLMC method.

2.4.2. MLCV: Examples with two levels. For illustrative purposes and clear exposure of several motivational concerns, we first introduce the main underlying concepts of our approach by considering a significantly simplified example. In particular, we consider only two levels, a coarser level $\ell-1$ and a finer level $\ell$. The backbone of MLMC is the hierarchical variance reduction technique, where statistical moments at level $\ell$ use simulations from coarser discretization level $q_{\ell-1}$ as a control variate with "known" $\mathbb{E}\left[q_{\ell-1}\right]$ and the predetermined coupling coefficient $\alpha_{\ell}=1$. The coupled statistical $Q_{0} I q_{\ell}^{*}$ with the same expected value as $q_{\ell}$ is given by

$$
q_{\ell}^{*}=q_{\ell}+\alpha\left(\mathbb{E}\left[q_{\ell-1}\right]-q_{\ell-1}\right) .
$$

The variance reduction that is achieved in (17) by considering random variable $q_{\ell}^{*}$ instead of $q_{\ell}$ depends on the correlation between $q_{\ell}$ and $q_{\ell-1}$,

$$
\mathbb{V}\left[q_{\ell}^{*}\right]=\mathbb{V}\left[q_{\ell}+\alpha\left(\mathbb{E}\left[q_{\ell-1}\right]-q_{\ell-1}\right)\right]=\mathbb{V}\left[q_{\ell}\right]+\alpha^{2} \mathbb{V}\left[q_{\ell-1}\right]-2 \alpha \operatorname{Cov}\left[q_{\ell}, q_{\ell-1}\right]
$$

Copyright (C) by SIAM. Unauthorized reproduction of this article is prohibited. 
Statistical expectation estimators $E_{M_{\ell}}[\cdot]$ using $M_{\ell}$ samples are applied to $q_{\ell}^{*}$ instead of $q_{\ell}$, leading to the building block of the MLMC estimator:

$$
\mathbb{E}\left[q_{\ell}\right]=\mathbb{E}\left[q_{\ell}^{*}\right] \approx E_{M_{\ell}, M_{\ell-1}}\left[q_{\ell}^{*}\right]=E_{M_{\ell}}\left[q_{\ell}\right]+\alpha\left(\mathbb{E}\left[q_{\ell-1}\right]-E_{M_{\ell}}\left[q_{\ell-1}\right]\right) .
$$

In standard MLMC, the expectation $\mathbb{E}\left[q_{\ell-1}\right]$ is further approximated independently by the estimate $E_{M_{\ell-1}}\left[q_{\ell-1}\right]$ with a different number of MC samples $M_{\ell-1}$, and the coefficient $\alpha$ in (17)-(19) is set to unity; see [24, 23]. This constraint on coefficient $\alpha$ could potentially, but not necessarily, limit the efficiency of the variance reduction technique. For instance, under the assumption (to expose the role of level correlation) that variances at both levels are comparable, i.e., $\mathbb{V}\left[q_{\ell}\right] \approx \mathbb{V}\left[q_{\ell-1}\right]$, the variance of (19) would increase provided the correlation coefficient Cor $\left[q_{\ell}, q_{\ell-1}\right]$ drops below a threshold of $1 / 2$,

$$
\mathbb{V}\left[q_{\ell}^{*}\right] \approx 2 \mathbb{V}\left[q_{\ell}\right]-2 \operatorname{Cor}\left[q_{\ell}, q_{\ell-1}\right] \mathbb{V}\left[q_{\ell}\right] \geq 2 \mathbb{V}\left[q_{\ell}\right]-2 \frac{1}{2} \mathbb{V}\left[q_{\ell}\right]=\mathbb{V}\left[q_{\ell}\right]
$$

To avoid this, the optimal $\alpha$ minimizing the variance of $q_{\ell}^{*}$ as in (18) can be used:

$$
\alpha=\frac{\operatorname{Cov}\left[q_{\ell}, q_{\ell-1}\right]}{\mathbb{V}\left[q_{\ell-1}\right]} \approx \operatorname{Cor}\left[q_{\ell}, q_{\ell-1}\right] .
$$

A consequence of (21) is that the predetermined choice of $\alpha=1$ in (17) is optimal only under very restrictive conditions: perfectly correlated levels with correlation coefficient $\operatorname{Cor}\left[q_{\ell}, q_{\ell-1}\right]=1$ and the assumption that coarser level estimates $\mathbb{E}\left[q_{\ell-1}\right]$ are already available (hence no computation is needed, i.e., $\mathbb{W}_{\ell-1}=0$ ). Note, that for optimal $\alpha$ as in (21), variance is always reduced in (18), even for $0<\operatorname{Cor}\left[q_{\ell}, q_{\ell-1}\right]<$ $1 / 2$,

$$
\mathbb{V}\left[q_{\ell}^{*}\right]=\left(1-\operatorname{Cor}\left[q_{\ell}, q_{\ell-1}\right]^{2}\right) \mathbb{V}\left[q_{\ell}\right]<\mathbb{V}\left[q_{\ell}\right]
$$

For $\mathbb{W}_{\ell-1} \neq 0$, it is necessary to obtain an estimate for $\mathbb{E}\left[q_{\ell-1}\right]$ in (17) as well. In such case, using the independence of estimators $E_{M_{\ell}}$ and $E_{M_{\ell-1}}$ and the central limit theorem, the variance of the two-level estimator $E_{M_{\ell}, M_{\ell-1}}\left[q_{\ell}^{*}\right]$ as in (19) with additional coarse-level approximation $E_{M_{\ell-1}}\left[q_{\ell-1}\right]$ is given by

$$
\mathbb{V}\left[E_{M_{\ell}, M_{\ell-1}}\left[q_{\ell}^{*}\right]\right]=\frac{\mathbb{V}\left[q_{\ell}-\alpha q_{\ell-1}\right]}{M_{\ell}}+\frac{\mathbb{V}\left[\alpha q_{\ell-1}\right]}{M_{\ell-1}}
$$

Minimizing the variance of the estimator $E_{M_{\ell}, M_{\ell-1}}\left[q_{\ell}^{*}\right]$ in (23), i.e., finding jointly optimal unknown parameters $\alpha, M_{\ell}, M_{\ell-1}$, is a particularly challenging problem (even for this simple two-level example), since $\alpha$ is a real number and $M_{\ell}, M_{\ell-1}$ are integers. Several existing results address this optimization problem by introducing empirical simplifications, such as performing a joint real-valued optimization of $\alpha$ and $M_{\ell}, M_{\ell-1}$ together and then rounding down $M_{\ell}, M_{\ell-1}$ to integers [60,61], or performing onlyinteger optimization on $M_{\ell}, M_{\ell-1}$ while disregarding $\alpha$ by using standard MLMC only [57]. On the other hand, even solving such a mixed optimization problem would result in an overall emprical sampling algorithm, since level variances and covariances required in (23) are not known and need to be estimated empirically. Motivated by these considerations, we instead propose a simple empirical optimization procedure for $(23)$, which only partially relies on the conventional Lagrange multiplier methodologies used for the standard MLMC in [24]. That is, instead of performing Lagrange 
multiplier optimization (over reals) jointly on $\alpha$ and $M_{\ell}, M_{\ell-1}$, we first optimize $\alpha$ independently of $M_{\ell}, M_{\ell-1}$ by introducing an auxiliary empirical variance reduction cost functional, and only then estimate $M_{\ell}, M_{\ell-1}$ using (also partially empirical due to rounding) Lagrange multipliers. To optimize $\alpha$ independently of $M_{\ell}, M_{\ell-1}$, we employ the level-specific inverse relation between the number of samples $M_{\ell}$ and the required computational cost per sample $\mathbb{W}_{\ell}$ :

$$
\frac{1}{M_{\ell}} \sim \mathbb{W}_{\ell}
$$

Using (24), we transform the variance estimate in (23) into an empirical total computational cost for variance reduction in $E_{M_{\ell}, M_{\ell-1}}\left[q_{\ell}^{*}\right]$,

$$
C\left[q_{\ell}^{*}\right]=\mathbb{V}\left[\alpha q_{\ell-1}\right] \mathbb{W}_{\ell-1}+\mathbb{V}\left[q_{\ell}-\alpha q_{\ell-1}\right] \mathbb{W}_{\ell},
$$

where variances of $\alpha q_{\ell-1}$ and $q_{\ell}-\alpha q_{\ell-1}$ are weighted by the corresponding computational costs $\mathbb{W}_{\ell-1}$ and $\mathbb{W}_{\ell}$, respectively. In order to find the empirically optimal $\alpha$, the computational variance reduction cost $C\left[q_{\ell}^{*}\right]$ in (25) is minimized instead of $\mathbb{V}\left[q_{\ell}^{*}\right]$ in (18). The resulting empirically optimal coefficient $\alpha$ can be obtained by solving the linear equation resulting from setting the derivative of the total computational cost for the variance reduction $C\left[q_{\ell}^{*}\right]$ with respect to $\alpha$ to zero:

(26)

$$
\begin{aligned}
\frac{\mathrm{d} C\left[q_{\ell}^{*}\right]}{\mathrm{d} \alpha} & =\frac{\mathrm{d}}{\mathrm{d} \alpha}\left(\alpha^{2} \mathbb{V}\left[q_{\ell-1}\right] \mathbb{W}_{\ell-1}+\left(\mathbb{V}\left[q_{\ell}\right]+\alpha^{2} \mathbb{V}\left[q_{\ell-1}\right]-2 \alpha \operatorname{Cov}\left[q_{\ell}, q_{\ell-1}\right]\right) \mathbb{W}_{\ell}\right)=0 \\
& \Leftrightarrow 2 \alpha \mathbb{V}\left[q_{\ell-1}\right] \mathbb{W}_{\ell-1}+2 \alpha \mathbb{V}\left[q_{\ell-1}\right] \mathbb{W}_{\ell}-2 \operatorname{Cov}\left[q_{\ell}, q_{\ell-1}\right] \mathbb{W}_{\ell}=0 \\
& \Leftrightarrow \alpha \mathbb{V}\left[q_{\ell-1}\right]\left(\mathbb{W}_{\ell-1}+\mathbb{W} \ell-\operatorname{Cov}\left[q_{\ell}, q_{\ell-1}\right] \mathbb{W}_{\ell}=0 .\right.
\end{aligned}
$$

The resulting linear equation (26) is then solved for the empirically optimal $\alpha$ (and its approximation provided $\left.\mathbb{V}\left[q_{\ell}\right] \approx \mathbb{V}\left[q_{\ell-1}\right]\right)$ :

$$
\alpha=\frac{\mathbb{W}_{\ell}}{\mathbb{W}_{\ell}+\mathbb{W}_{\ell-1}} \frac{\operatorname{Cov}\left[q_{\ell}, q_{\ell-1}\right]}{\mathbb{V}\left[q_{\ell-1}\right]} \approx \frac{\mathbb{W}_{\ell}}{\mathbb{W}_{\ell}+\mathbb{W}_{\ell-1}} \operatorname{Cor}\left[q_{\ell}, q_{\ell-1}\right]
$$

We note, that (27) reduces to the standard control variate coefficient (21) if $\mathbb{W}_{\ell-1}=0$.

2.4.3. MLCV: Control variates for MLMC. We generalize the concept of empirically optimal control variate coefficients from subsubsection 2.4 .2 to an arbitrary number of levels in the MLMC estimator. In particular, control variate contributions from multiple levels can be used,

(28) $q_{L}^{*}=\left(q_{L}-\alpha_{L-1} q_{L-1}\right)+\left(\alpha_{L-1} q_{L-1}-\alpha_{L-2} q_{L-2}\right)+\cdots+\left(\alpha_{1} q_{1}-\alpha_{0} q_{0}\right)+\alpha_{0} q_{0}$,

allowing for a generalization of the telescoping sum in (13) to the control variate setting introduced in (17), where the coefficient for the finest level $\ell=L$ is fixed to $\alpha_{L}=1$ :

$$
\mathbb{E}\left[q_{L}\right]=\mathbb{E}\left[q_{L}^{*}\right]=\alpha_{0} \mathbb{E}\left[q_{0}\right]+\sum_{\ell=1}^{L}\left(\alpha_{\ell} \mathbb{E}\left[q_{\ell}\right]-\alpha_{\ell-1} \mathbb{E}\left[q_{\ell-1}\right]\right)
$$

Using the mutual statistical independence of $\alpha_{0} q_{0}$ and differences $\alpha_{\ell} q_{\ell}-\alpha_{\ell-1} q_{\ell-1}$ together with the central limit theorem analogously to subsubsection 2.4.2, the empirical total computational variance reduction cost employing (24) and generalizing

Copyright (C) by SIAM. Unauthorized reproduction of this article is prohibited. 
(25) for $q_{L}^{*}$ over all levels as in (28), is given by

$$
\begin{aligned}
C\left[q_{L}^{*}\right] & =\mathbb{V}\left[\alpha_{0} q_{0}\right] \mathbb{W}_{0}+\sum_{\ell=1}^{L} \mathbb{V}\left[\alpha_{\ell} q_{\ell}-\alpha_{\ell-1} q_{\ell-1}\right] \mathbb{W}_{\ell} \\
& =\alpha_{0}^{2} \mathbb{V}\left[q_{0}\right] \mathbb{W}_{0}+\sum_{\ell=1}^{L}\left(\alpha_{\ell}^{2} \mathbb{V}\left[q_{\ell}\right]+\alpha_{\ell-1}^{2} \mathbb{V}\left[q_{\ell-1}\right]-2 \alpha_{\ell} \alpha_{\ell-1} \operatorname{Cov}\left[q_{\ell}, q_{\ell-1}\right]\right) \mathbb{W}_{\ell} .
\end{aligned}
$$

Minimization of $C\left[q_{L}^{*}\right]$ pertains to solving a linear system of equations,

$$
\frac{\partial}{\partial \alpha_{\ell}} C\left[q_{L}^{*}\right]=0, \quad \ell=0, \ldots, L-1 .
$$

Using the following abbreviations,

$$
\begin{aligned}
\sigma_{\ell}^{2} & =\mathbb{V}\left[q_{\ell}\right], \\
\mathbb{W}_{\ell, \ell-1} & =\mathbb{W}_{\ell}+\mathbb{W}_{\ell-1}, \\
\sigma_{\ell, \ell-1}^{2} & =\operatorname{Cov}\left[q_{\ell}, q_{\ell-1}\right], \\
\mathbf{a} & =\left(\alpha_{0}, \ldots, \alpha_{L-1}\right)^{\top} \in \mathbb{R}^{L},
\end{aligned}
$$

the linear system in (31) can be written in the tridiagonal matrix form

$$
\mathrm{Ma}=\mathrm{b}
$$

with the load vector given by

$$
\mathbf{b}=(\underbrace{0, \ldots, 0}_{L-1}, \sigma_{L, L-1}^{2} \mathbb{W})^{\top} \in \mathbb{R}^{L}
$$

and the tridiagonal matrix $\mathbf{M} \in \mathbb{R}^{L \times L}$ given by

$$
\mathbf{M}=\left[\begin{array}{cccc}
\sigma_{0}^{2} \mathbb{W}_{1,0} & -\sigma_{1,0}^{2} \mathbb{W}_{1} & & \\
-\sigma_{1,0}^{2} \mathbb{W}_{1} & \ddots & \ddots & \\
& \ddots & \ddots & -\sigma_{L-1, L-2}^{2} \mathbb{W}_{L-1} \\
& & -\sigma_{L-1, L-2}^{2} \mathbb{W}_{L-1} & \sigma_{L-1}^{2} \mathbb{W}_{L, L-1}
\end{array}\right] .
$$

For a special case of two levels with $L=1$, the solution of (33) reduces to (27). In the general multilevel setting with $L \geq 2$, the well-posedness (existence and uniqueness of the solution) of the system (33) is guaranteed by the following theorem.

THEOREM 2.1 (well-posedness of the control variate coefficients). If $\mathbb{W}_{\ell}, \sigma_{\ell}^{2}>0$ for every level $\ell=0, \ldots, L$ then matrix $\mathbf{M}$ in (35) is positive-definite and hence the system (33) can be solved uniquely for the control variate coefficients vector a.

Copyright (C) by SIAM. Unauthorized reproduction of this article is prohibited. 
Proof. First, we verify that $\mathbf{M}$ in (35) is positive-definite, i.e., that $\mathbf{a}^{\top} \mathrm{Ma}>0$ for all $\mathrm{a} \neq 0$. In particular, the matrix-vector multiplications expand to

$$
\begin{aligned}
\mathbf{a}^{\top} \mathbf{M a}= & \alpha_{0}^{2} \sigma_{0}^{2} \mathbb{W}_{1}+\alpha_{0}^{2} \sigma_{0}^{2} \mathbb{W}_{0}-\alpha_{1} \alpha_{0} \sigma_{1,0}^{2} \mathbb{W}_{1} \\
& -\alpha_{1} \alpha_{0} \sigma_{1,0}^{2} \mathbb{W}_{1}+\alpha_{1}^{2} \sigma_{1}^{2} \mathbb{W}_{2}+\alpha_{1}^{2} \sigma_{1}^{2} \mathbb{W}_{1}-\alpha_{2} \alpha_{1} \sigma_{2,1}^{2} \mathbb{W}_{2} \\
& \cdots \\
& -\alpha_{\ell} \alpha_{\ell-1} \sigma_{\ell, \ell-1}^{2} \mathbb{W}_{\ell}+\alpha_{\ell}^{2} \sigma_{\ell}^{2} \mathbb{W}_{\ell+1}+\alpha_{\ell}^{2} \sigma_{\ell}^{2} \mathbb{W}_{\ell}-\alpha_{\ell+1} \alpha_{\ell} \sigma_{\ell+1, \ell}^{2} \mathbb{W}_{\ell+1} \\
& \cdots \\
& -\alpha_{L-2} \alpha_{L-3} \sigma_{L-2, L-3}^{2} \mathbb{W}_{L-2}+\alpha_{L-2}^{2} \sigma_{L-2}^{2} \mathbb{W}_{L-1}+\alpha_{L-2}^{2} \sigma_{L-2}^{2} \mathbb{W}_{L-2} \\
& -\alpha_{L-1} \alpha_{L-2} \sigma_{L-1, L-2}^{2} \mathbb{W}_{L-1}+\alpha_{L-1}^{2} \sigma_{L-1}^{2} \mathbb{W}_{L}+\alpha_{L-1}^{2} \sigma_{L-1}^{2} \mathbb{W}_{L-1} .
\end{aligned}
$$

In the expansion (36) of $\mathbf{a}^{\top} \mathrm{Ma}$, for every level $\ell=1, \ldots, L-1$, each group of terms with identical common factors $\mathbb{W}_{\ell}$ adds up to a nonnegative sum $\Sigma_{\ell}$, defined by

$$
\begin{aligned}
\Sigma_{\ell} & =\alpha_{\ell-1}^{2} \sigma_{\ell-1}^{2} \mathbb{W}_{\ell}-\alpha_{\ell} \alpha_{\ell-1} \sigma_{\ell, \ell-1}^{2} \mathbb{W}_{\ell}-\alpha_{\ell} \alpha_{\ell-1} \sigma_{\ell, \ell-1}^{2} \mathbb{W}_{\ell}+\alpha_{\ell}^{2} \sigma_{\ell}^{2} \mathbb{W}_{\ell} \\
& =\mathbb{W}_{\ell}\left(\alpha_{\ell-1}^{2} \sigma_{\ell-1}^{2}-2 \alpha_{\ell} \alpha_{\ell-1} \sigma_{\ell, \ell-1}^{2}+\alpha_{\ell}^{2} \sigma_{\ell}^{2}\right) \\
& =\mathbb{W}_{\ell}\left(\alpha_{\ell-1}^{2} \mathbb{V}\left[q_{\ell-1}\right]-2 \alpha_{\ell} \alpha_{\ell-1} \operatorname{Cov}\left[q_{\ell}, q_{\ell-1}\right]+\alpha_{\ell}^{2} \mathbb{V}\left[q_{\ell}\right]\right) \\
& =\mathbb{W}_{\ell} \mathbb{V}\left[\alpha_{\ell-1} q_{\ell-1}-\alpha_{\ell} q_{\ell}\right] \geq 0 .
\end{aligned}
$$

Employing inequality (37), the expansion of $\mathbf{a}^{\top} \mathbf{M a}$ in (36) can be bounded from below by the remaining terms involving factors $\mathbb{W}_{0}$ and $\mathbb{W}_{L}$, which are both nonnegative, since $\mathbb{W}_{0}$ and $\mathbb{W}_{L}$ were both assumed to be positive:

$$
\mathbf{a}^{\top} \mathbf{M a} \geq \alpha_{0}^{2} \sigma_{0}^{2} \mathbb{W}_{0}+\alpha_{L-1}^{2} \sigma_{L-1}^{2} \mathbb{W}_{L} \geq 0
$$

We note that strict equalities in (37) and (38) can be achieved if and only if all coefficients $\alpha_{\ell}$ are zero, i.e., $\mathbf{a}=0$. This proves that $\mathbf{a}^{\top} \mathrm{Ma}>0$ for all $\mathrm{a} \neq 0$ and, hence, matrix $\mathrm{M}$ in (35) is positive-definite. Since positive-definite matrices are invertible, the system of equations (33) can be solved uniquely.

Generalizing (14), the unbiased MLCV estimator is then given by

$$
\mathbb{E}\left[q_{L}\right] \approx E^{\mathrm{MLCV}}\left[q_{L}\right]=\alpha_{0} \frac{1}{M_{0}} \sum_{i=1}^{M_{0}} q_{0}^{i}+\sum_{\ell=1}^{L} \frac{1}{M_{\ell}} \sum_{i=1}^{M_{\ell}}\left(\alpha_{\ell} q_{\ell}^{i}-\alpha_{\ell-1} q_{\ell-1}^{i}\right)
$$

with empirically optimal control variate coefficients $\alpha_{\ell}$ given by (33). We assume that samples for $\alpha_{0} q_{0}^{i}$ and differences $\alpha_{\ell} q_{\ell}^{i}-\alpha_{\ell-1} q_{\ell-1}^{i}$ are drawn independently (however, individual $q_{\ell}^{i}$ and $q_{\ell-1}^{i}$ are statistically dependent). The statistical mean square error $\varepsilon_{\text {MLCV }}^{2}$ of the MLCV estimator is given by the sum of sample-reduced variances of $\alpha_{\ell}$-weighted differences between every two consecutive levels,

$$
\begin{aligned}
\varepsilon_{\mathrm{MLCV}}^{2}=\mathbb{E}\left[\left(E^{\mathrm{MLCV}}\left[q_{L}\right]-\mathbb{E}\left[q_{L}\right]\right)^{2}\right] & =\frac{\mathbb{V}\left[\alpha_{0} q_{0}\right]}{M_{0}}+\sum_{\ell=1}^{L} \frac{\mathbb{V}\left[\alpha_{\ell} q_{\ell}-\alpha_{\ell-1} q_{\ell-1}\right]}{M_{\ell}} \\
& =: \frac{\tilde{\sigma}_{0}^{2}}{M_{0}}+\sum_{\ell=1}^{L} \frac{\tilde{\sigma}_{\ell}^{2}}{M_{\ell}} .
\end{aligned}
$$

Copyright (C) by SIAM. Unauthorized reproduction of this article is prohibited. 
Given computational costs $\mathbb{W}_{\ell}$ of evaluating a single approximation $q_{\ell}^{i}$ for each level $\ell=0, \ldots, L$ and a desired tolerance $\tau>0$, the total computational cost of MLCV can be minimized under the constraint of $\varepsilon_{\text {MLCV }}^{2} \leq \tau$ by choosing the optimal (up to rounding to integers) number of $\mathrm{MC}$ samples $M_{\ell}$ on each level according to [52], yielding

$$
M_{\ell}=\left\lceil\frac{1}{\tau^{2}} \sqrt{\frac{\tilde{\sigma}_{\ell}^{2}}{\mathbb{W}_{\ell}}} \sum_{k=0}^{L} \sqrt{\tilde{\sigma}_{k}^{2} \mathbb{W}_{k}}\right\rceil
$$

Alternatively, given the available total computational budget $\mathcal{B}$ instead of a desired tolerance $\tau$, the MLCV error $\varepsilon_{\mathrm{MLCV}}^{2}$ is minimized by choosing the optimal (up to rounding to integers) $M_{\ell}$ according to

$$
M_{\ell}=\left\lceil\mathcal{B} \sqrt{\frac{\tilde{\sigma}_{\ell}^{2}}{\mathbb{W}_{\ell}}} / \sum_{k=0}^{L} \sqrt{\tilde{\sigma}_{k}^{2} \mathbb{W}_{k}}\right\rceil .
$$

A discrete optimization problem for (40) could be formulated, avoiding roundoff operations in (41) or (42), and providing a truly optimal integer $M_{\ell}$, as suggested in [57]. We note, however, that even though such rounding does not influence the efficiency of the method on coarser levels, where the number of samples is large, and are most probably overshadowed by the used approximate estimators for $\tilde{\sigma}_{\ell}^{2}$, such empirical rounding could have detrimental effects on computational efficiency on the finest levels of resolution. In particular, the rounding of samples can be particularly detrimental to the optimal performance of MLMC coupled with very expensive HPC codes, since the largest impact of rounding is on the highest resolution levels, where the number of samples is expected to be very low (as observed in the numerical simulations in section 3), provided a sufficient number of levels is used [57].

2.4.4. Adaptive MLCV algorithm. The MLCV algorithm proceeds iteratively, with each iteration improving the accuracy of the estimated statistics for the QoIs, such as the expectation $\mathbb{E}[q]$. Each iteration improves the accuracy of auxiliary parameters, such as $\sigma_{\ell}^{2}, \sigma_{\ell, \ell-1}^{2}, \mathbb{W}_{\ell}, \tilde{\sigma}_{\ell}^{2}$ and, hencforth, also the optimality of the derived quantities, such as control variate coefficients $\alpha_{\ell}$ and the number of samples $M_{\ell}$, for each level $\ell=0, \ldots, L$.

A single iteration of the algorithm consists of the following 8 steps.

(1) Warm-up: Begin with a level-dependent number of warm-up samples. The optimal choice is dependent on the specific application; we used a heuristic choice

$$
M_{\ell}=\left\lceil\frac{\mathbb{W}_{L}}{\mathbb{W}_{\ell}} \frac{1}{2^{(L-\ell)}}\right\rceil, \quad \ell=L, L-1, \ldots, 0 .
$$

The choice of $M_{\ell}$ as in (43) prevents efficiency deterioration of the final MLCV estimator by ensuring that the total computational budget for the warm-up iteration does not exceed $2 \mathbb{W}_{L}$ (i.e., comparable to the computational work required for a single simulation on the finest resolution level); at the same time, it allows one to prescribe sufficiently many samples on the coarser levels, where $\tilde{\sigma}_{\ell}^{2}$ is expected to be large. In our particular application, computational work for each level scales as $\mathbb{W}_{\ell}=\mathcal{O}\left(2^{4 \ell}\right)$ and, hence, the number of required warm-up samples is given by $1,8, \ldots, 2^{3 L}$ for $\ell=L, L-1, \ldots, 0$. We note

Copyright (C) by SIAM. Unauthorized reproduction of this article is prohibited. 
that a constant (level-independent) number of warm-up samples can be very inefficient $[52,16]$.

(2) Solver: Evaluate approximations $q_{\ell}^{i}$ for each level $\ell=0, \ldots, L$ and sample $i=1, \ldots, M_{\ell}$, which have not yet been evaluated in previous iterations.

(3) Indicators: Using $q_{\ell}^{i}$, estimate $\sigma_{\ell}^{2}, \sigma_{\ell, \ell-1}^{2}$, and $\mathbb{W}_{\ell}$ for $\ell=0, \ldots, L$. Optionally, empirical estimates of $\sigma_{\ell, \ell-1}^{2}$ could be used within a Bayesian inference framework to fit an exponential decay model for $\sigma_{\ell, \ell-1}^{2}$ w.r.t. levels $\ell=1, \ldots, L-1$. Assuming Gaussian errors, this reduces to a least-squares line fit to the natural logarithm of indicators $\sigma_{\ell, \ell-1}^{2}$.

(4) Coefficients: Compute the control variate coefficients $\alpha_{\ell}$ from estimated $\sigma_{\ell}^{2}$ and $\sigma_{\ell, \ell-1}^{2}$ using (33).

(5) Errors: Using $q_{\ell}^{i}$ and $\alpha_{\ell}$, estimate $\alpha_{\ell}$-weighted covariances $\tilde{\sigma}_{\ell}^{2}$ and total sampling error $\hat{\varepsilon}_{\mathrm{MLCV}}^{2} \approx \varepsilon_{\mathrm{MLCV}}^{2}$ as in $(40)$.

(6) Estimator: If the required tolerance is reached, i.e., $\hat{\varepsilon}_{\mathrm{MLCV}} \leq \tau$, or if the prescribed computational budget $\mathcal{B}$ is spent, then evaluate the MLCV estimator (39) and terminate the algorithm. Otherwise, proceed to the optimization step.

(7) Optimization: Compute the optimal (up to rounding to integers) required number of samples $\hat{M}_{\ell}$ from $\tilde{\sigma}_{\ell}$ and $\mathbb{W}_{\ell}$ using either (41) or (42), respectively. Remark. If we obtain $\hat{M}_{\ell}<M_{\ell}$ for some level $\ell$, we keep the already computed samples, i.e., we set $\hat{M}_{\ell}=M_{\ell}$. In order to adjust for such a constraint in the optimization problem, we subtract the corresponding sampling error $\tilde{\sigma}_{\ell}^{2} / M_{\ell}$ from the required tolerance $\tau^{2}$, or subtract the corresponding amount of computational budget $M_{\ell} \mathbb{W}_{\ell}$ from $\mathcal{B}$, respectively. Afterwards, the number of samples $\hat{M}_{\ell}$ for the remaining levels (where $\hat{M}_{\ell}=M_{\ell}$ was not enforced) are reoptimized according to either (41) or (42), respectively. We repeat this procedure until $\hat{M}_{\ell} \geq M_{\ell}$ is satisfied for all levels $\ell=0, \ldots, L$.

(8) Iterations: Go back to step (2) and continue the algorithm with the updated number of samples $\hat{M}_{\ell}$.

If the empirical estimates in steps (3)-(5) of the above adaptive MLCV algorithm are sufficiently accurate, the algorithm will terminate after two iterations - the initial warm-up iteration and one additional iteration with already empirically optimal $\alpha_{\ell}$ and $M_{\ell}$. A more detailed discussion of the special cases within the MLCV algorithm, alternative approaches, related work, and possible extensions is provided in subsection 2.5. We would also like to note, that same samples are used to estimate both the indicators in step (3) and the MLMC estimator in step (6). This could potentially introduce correlations between the estimated MLMC expectation and the number of used samples on each level, leading to an additional bias in the MLMC estimator. One way to avoid this would be to use different samples for indicators and for MLMC estimates, incurring additional computational overhead.

Alternative optimal coefficients (jointly with empirically rounded $M_{\ell}$ to integers) for each level in the MLMC estimator was suggested in [59, 61], where a multifidelity MC method is described. There, some statistical realizations (samples) are reused, i.e., the same result is used in both estimates $E_{M_{\ell}}\left[\alpha_{\ell} q_{\ell}\right]$ and $-E_{M_{\ell+1}}\left[\alpha_{\ell} q_{\ell}\right]$, each contributing to a separate difference in the telescoping MLMC estimator (39). Such "recycling" strategy requires less sampling, however, error analysis complexity is highly increased due to additional statistical dependencies, absent in the MLCV method. On the other hand, for "recycled" sampling, the resulting error minimization problem is separable in terms of coefficients $\alpha_{\ell}$ and number of samples $M_{\ell}$ and, hence, no linear system

Copyright (C) by SIAM. Unauthorized reproduction of this article is prohibited. 
(33) needs to be solved [59]. The linear system (33) is, however, very small, sparse, and needs to be solved only a few times, hence, is not a bottleneck of this algorithm.

We do not have at present a comparative study to evaluate which approach could provide better variance reduction (in particular when taking into account the required computational work). On one hand, we could have a joint optimization using Lagrange multipliers followed by the rounding of $M_{\ell}$ (analogous to the initial work in [25]) and, on the other, the proposed MLCV employing a simplified empirical variance reduction cost functional (30) taking into account different computational costs on different levels of resolution.

2.5. Technical considerations regarding iterations in the MLCV algorithm. In this section we briefly outline technicalities that are often encountered in practical use of the MLCV algorithm from subsubsection 2.4.4, namely, the strategies to avoid inefficient oversampling at the finest resolution level during the "warm-up" step 0 and to efficiently mitigate uncertainties due to possible inaccurate indicator estimates in the algorithm execution control at step 6.

2.5.1. The number of warm-up samples on the finest resolution level. Notice, that in order to have empirical estimates of $\sigma_{\ell}^{2}, \sigma_{\ell, \ell-1}^{2}$, and $\tilde{\sigma}_{\ell}^{2}$, at least two samples would be required on each level $\ell=0, \ldots, L$. Enforcing $M_{L} \geq 2$ might be very inefficient in the cases when only one sample is actually needed, since in presently considered applications the most computationally expensive samples are actually at the finest level $\ell=L$. To avoid this, initially only one warm-up sample on the finest mesh level $\ell=L$ could be computed, i.e., $M_{L}=1$. For subsequent optimization steps of each $M_{\ell}$, the variance of level difference $\sigma_{\ell, \ell-1}^{2}$ for level $\ell=L$ is inferred from available measurements on lower resolution levels using Bayesian inference, as described in step (3) of the MLCV algorithm. If more than one sample is actually required on the finest resolution level, optimization step (7) of the adaptive MLCV algorithm above will adjust $M_{L}$ to the correct empirically optimal value and additional empirical estimates would be available for even more accurate inference.

2.5.2. Control of adaptive MLCV iterations for inaccurate indicators. Final MLCV error $\varepsilon_{\text {MLCV }}$ could be underestimated by $\hat{\varepsilon}_{M L C V}$ in step 5 and be actually larger than the prescribed tolerance $\tau$, since we only ensure the estimated total error $\hat{\varepsilon}_{\mathrm{MLCV}} \leq \tau$. Since $\hat{\varepsilon}_{\mathrm{MLCV}}$ is based on the randomized statistical estimators, it is also random and has a spread around its mean $\mathbb{E}\left[\hat{\varepsilon}_{\mathrm{MLCV}}\right]=\varepsilon_{\mathrm{MLCV}}$. We note that probability $\mathbb{P}\left[\varepsilon_{\mathrm{MLCV}}>\hat{\varepsilon}_{\mathrm{MLCV}}\right]$ of the resulting MLMC error $\varepsilon_{\mathrm{MLCV}}$ being larger than the estimated error $\hat{\varepsilon}_{\mathrm{MLCV}}$ (which is forced to be smaller than the prescribed tolerance $\tau$ ) can be reduced by sufficiently increasing the number of samples $\hat{M}_{\ell}$ according to the estimates of the fourth centered moment (kurtosis) $\kappa_{\ell}^{2}$ of the level differences. Such estimates would provide the variance estimates $\hat{\kappa}_{\ell}^{2} \approx \mathbb{E}\left[\left(\hat{\sigma}_{\ell}^{2}\right)^{2}-\mathbb{E}\left[\hat{\sigma}_{\ell}^{2}\right]^{2}\right]$ of the empirical variance estimators $\hat{\sigma}_{\ell}^{2}$. Then, increasing $\hat{M}_{\ell}$ by several standard deviations $\hat{\kappa}_{\ell}$ of $\hat{\sigma}_{\ell}^{2}$, the required percentile level $\alpha$ of the confidence interval $[\alpha / 2,1-\alpha / 2]$ of $\hat{\varepsilon}_{\mathrm{MLCV}}$ can be reduced below the prescribed tolerance $\tau$, this way ensuring the required high probability $\mathbb{P}[\varepsilon \leq \tau] \geq 1-\alpha$ of true MLMC error $\varepsilon$ not exceeding the prescribed tolerance $\tau$. A continuation MLMC method incorporating similar techniques for estimation and control of error confidence intervals was already proposed in [16]. However, updating estimates after each computed sample could be very inefficient for large HPC applications, since such incremental techniques require heavy synchronization and would make efficient load balancing on distributed many-core systems very challenging. For such applications, only a small number of MLCV iterations is desired, which is the

Copyright (C) by SIAM. Unauthorized reproduction of this article is prohibited. 


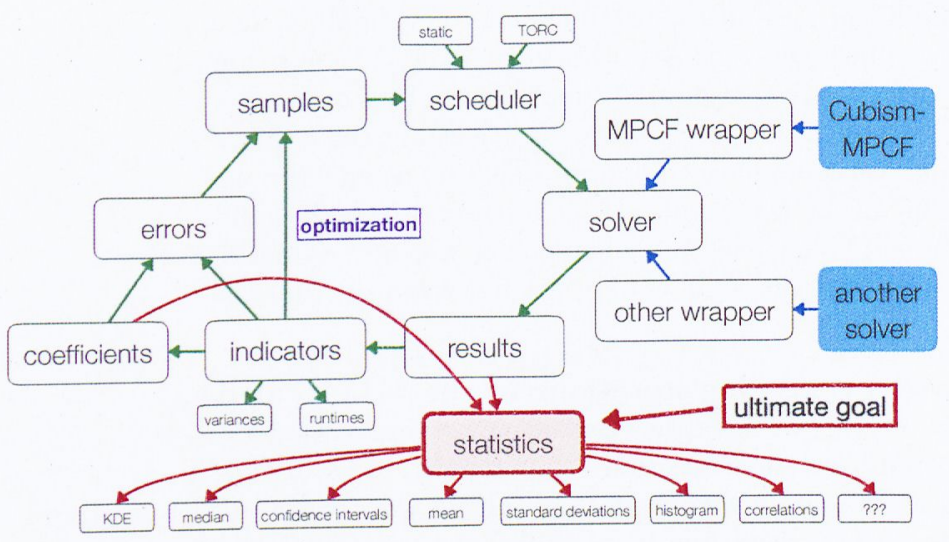

FIG. 3. Scheme of the MLCV implementation library PyMLMC.

case if indicator estimates from the initial warm-up step 1 are sufficiently accurate and the termination criteria in step 6 are implemented only approximately (for instance, with a small allowed $5 \%$ deviation margin). Analogous considerations are valid when the optimization of samples is based on the total computational budget (i.e., (42)) rather than desired tolerance.

2.6. PyMLMC. The MLCV algorithm is distributed as an open source library PyMLMC [74]. A diagram of the software components is shown in Figure 3. PyMLMC provides a modular framework for native execution of deterministic solvers in their respective "sandbox" directories. This allows maximum flexibility for programming languages, distributed and/or shared memory architectures, and support for manycore accelerators. Due to the lack of communication among such sandboxed executions for each sample, the load balancing across samples can be relayed to the submission system (e.g., Slurm, LSF, LoadLeveler, Cobalt) of the compute cluster. Nested (across and within samples) parallelism is used, where few large parallel jobs are submitted for fine levels and, in parallel, many small (possibly even serial) jobs are submitted for coarse levels. To increase the efficiency and reduce the stress on submission systems, job batching (submitting a single job that computes multiple samples subsequently) and job merging (submitting a single job that computes multiple samples concurrently) or a combination of both is implemented. Once all samples (or at least some of them) are computed, statistical estimators are constructed as a postprocessing step using the NumPy and SciPy libraries. The "sandboxing" framework enables any additional required statistical estimates or QoIs to be evaluated at a later stage without the need to reexecute any of the computationally expensive sampling runs. The amount of required disk space in multilevel methods scales linearly w.r.t. the amount of the required computational budget. In particular, the total required disk space for all samples on all levels is of the same order as a single statistical estimator on the full three-dimensional domain. Hence, it is highly advantageous to keep all computed samples for the aforementioned postprocessing flexibility purposes.

In the present work (see section 3), we verified the efficiency of the (nested) parallelization of the MLCV coupled with the Cubism-MPCF solver on the entire MIRA supercomputer (Argonne National Laboratory) consisting of half a million cores. We note that large (exa)scale simulations on massively parallel computing 
platforms are subject to processor failures at run-time [12]. Exploiting the natural fault tolerance in MLCV-FVM due to independent sampling, a fault tolerant (FT) MLMC (FT-MLMC) method was implemented in [58] and was shown to perform in agreement with theoretical analysis in the presence of simulated, compound Poisson distributed, random hard failures of compute cores. Such FT mechanisms are also available in PyMLMC, and have successfully excluded one corrupted sample on the coarsest level in the simulations reported in section 3.

3. Numerical simulations and analysis. The initialization of the cavities employs experimental findings indicating a log-normal distribution for their radii [51], whereas the position vectors are generated according to a uniform distribution as there is no prior knowledge.

3.1. Spherical cloud of 500 gas cavities with log-normally distributed radii. For a cubic domain $D=[0 \mathrm{~mm}, 100 \mathrm{~mm}]^{3}$, we consider a cloud of 500 bubbles located at the center $(50 \mathrm{~mm}, 50 \mathrm{~mm}, 50 \mathrm{~mm})^{\top}$ of the domain with a radius of $R_{\text {cloud }}=20 \mathrm{~mm}$. The log-normal distribution for the radii of the cavity is clipped so as to contain bubbles only within the range of $r_{\min }=0.8 \mathrm{~mm}$ to $r_{\max }=1.2 \mathrm{~mm}$. The resulting cloud gas volume content (w.r.t. to the volume of the sphere with radius $R_{\text {cloud }}$ ) is approximately $5 \%$ and the resulting cloud interaction parameter $\beta$ is approximately 3 , where $\beta=\alpha\left(\frac{R_{\text {cloud }}}{R_{\text {avg }}}\right)^{2}$ with cloud gas volume fraction $\alpha$, cloud radius $R_{\text {cloud, }}$, and average cavity radius $R_{\text {avg }}$ (refer to [10] for a derivation). We note that both of these quantities depend on a statistical realization of the random cloud. An illustration of the cloud geometry is shown in Figure 4.

The cloud is initially at pressure equilibrium with the surrounding water phase at $p_{2,0}=0.5 \mathrm{MPa}$. Throughout the entire domain, the density of the gas phase is set to $\rho_{2,0}=5 \mathrm{~kg} / \mathrm{m}^{3}$ and the density of the liquid is set to $\rho_{1,0}=1,000 \mathrm{~kg} / \mathrm{m}^{3}$. Starting $1 \mathrm{~mm}$ away from the surface of the cloud, there is a smooth pressure increase towards the prescribed ambient pressure of $p_{\infty}=10 \mathrm{MPa}$, following the setup proposed in [76]. The resulting pressure ratio is $p_{\infty} / p_{2,0}=20$. At the boundaries, nonreflecting, characteristic-based boundary conditions are applied, together with a penalty term for the prescribed far-field pressure of $p_{\infty}=10 \mathrm{MPa}$ [64]. A single statistical realization
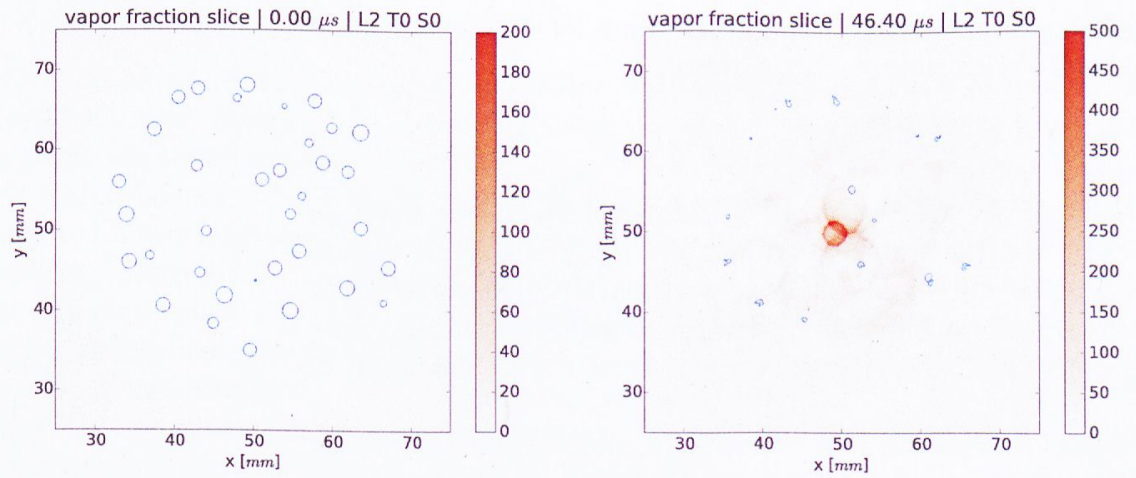

FIG. 4. Single statistical realization (sample) of the pressure field and cavity interfaces in the two-dimensional slice at $z=50 \mathrm{~mm}$ at the initial (left, $t=0 \mu \mathrm{s}$ ) and at the collapse (right, $t=46.4 \mu \mathrm{s}$ ) stages. Cavities collapse inwards, emitting pressure waves that focus near the center of the cloud. There is a two orders of magnitude difference in pressures at initial and collapse stages. 
(sample) of the pressure field and cavity interfaces across the two-dimensional slice at $z=50 \mathrm{~mm}$ computed using Cubism-MPCF with resolution of $1024^{3}$ mesh cells is depicted in Figure 4. Cavities are observed to collapse inwards, emitting pressure waves that focus near the center of the cloud at time $t=46.4 \mu \mathrm{s}$. We emphasize that each collapsing cavity emits a pressure shock wave (with propagating fronts depicted in Figure 4), leading to a low regularity (smoothness) of the approximated solution.

We consider four levels of spatial and temporal resolutions. The coarsest mesh consists of $512^{3}$ cells with two intermediate meshes of $1,024^{3}$ and $2,048^{3}$ resolutions, and the finest mesh with $4,096^{3}$ cells. The time step size decreases according to a prescribed CFL condition with CFL number set to 0.3 , resulting in approximately 2,250 and 20,000 time steps for the coarsest and finest levels, respectively. We note, that even on the coarsest resolution level, all cavities are resolved using at least approximately 5 mesh cells per radius. The convergence behavior of the CubismMPCF code for a single cavity analogous to cavities in these clouds was investigated in a recent work; see [66]. Based on this study, we expect strong bias in pressure sensors, whereas bias in vapor fraction sensors should be insignificant.

We note that the number of uncertainty sources in this simulation is very large: for each realization of a cloud, random three-dimensional spatial coordinates together with a random positive radius for all 500 cavities are needed, comprising in total 2,000 independent sources of uncertainty.

For each statistical sample of a collapsing cloud configuration and on each resolution level, simulations were performed for approximately $70 \mu \mathrm{s}$ in physical time. Depending on the random configuration of the cloud, the main collapse occurred at approximately $40-50 \mu \mathrm{s}$, followed by rebound and relaxation stages after $50 \mu \mathrm{s}$. The obtained results are discussed in the following sections.

3.2. Performance of the MLCV. We quantify the computational gains of the MLCV method by comparing it to standard MLMC and plain MC sampling methods. The chosen quantity of interest $q$ for (29) is the pressure as sampled by a sensor $p_{\mathbf{c}}$ placed at the center of the cloud and emulated as

$$
p_{\mathbf{c}}(t)=\frac{1}{\left|\mathcal{B}_{r}(\mathbf{c})\right|} \int_{\mathcal{B}_{r}(\mathbf{c})} p(\mathbf{x}, t) d \mathbf{x}
$$

where pressure $p$ is averaged over a sphere $\mathcal{B}_{r}(\mathbf{c})$ around the center of the cloud located at $\mathrm{c}=(50 \mathrm{~mm}, 50 \mathrm{~mm}, 50 \mathrm{~mm})^{\top}$ with radius $r=0.5 \mathrm{~mm}$,

$$
\mathcal{B}_{r}(\mathbf{c})=\left\{\mathrm{x} \in D:\|\mathrm{x}-\mathrm{c}\|_{2} \leq r\right\} .
$$

For this particular choice of the QoI, $q=p_{\mathrm{c}}$, estimated correlations between levels, implicitly used in (33), and the resulting empirically optimal control variate coefficients from (33) are depicted in Figure 5. Due to relatively high correlations between resolution levels, empirically optimal control variate coefficients exhibit only moderate deviations from unity, with the largest being at level 1 with deviation of $30 \%$.

Estimated variances of level differences, required in (33), and sampling errors for each level, computed in (40), are depicted in Figure 6. Variances of differences are decreasing for finer levels of resolution, which requires a smaller number of MC samples $M_{\ell}$ in order to reduce the statistical error on finer resolution levels, where sampling entails computationally very expensive numerical simulations. Measurements of variances of differences $\sigma_{\ell, \ell-1}^{2}$ are plotted as circles, with the associated error bars,

Copyright (C) by SIAM. Unauthorized reproduction of this article is prohibited. 

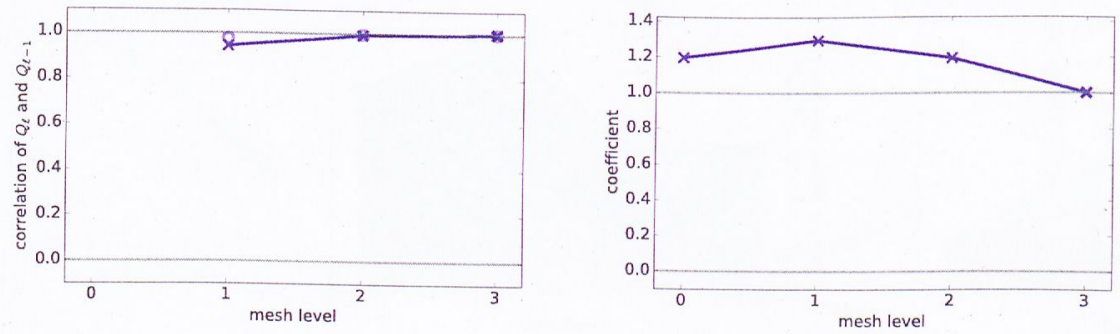

FIG. 5. Estimated correlations between levels (left) and the resulting empirically optimal control variate coefficients (right) as in (33). In the left plot, circles depict measurements of correlations with the associated error bars, and the solid line depicts the resulting inferred values. Relatively high correlations and moderate deviations (up to 30\%) in empirically optimal control variate coefficients are observed.
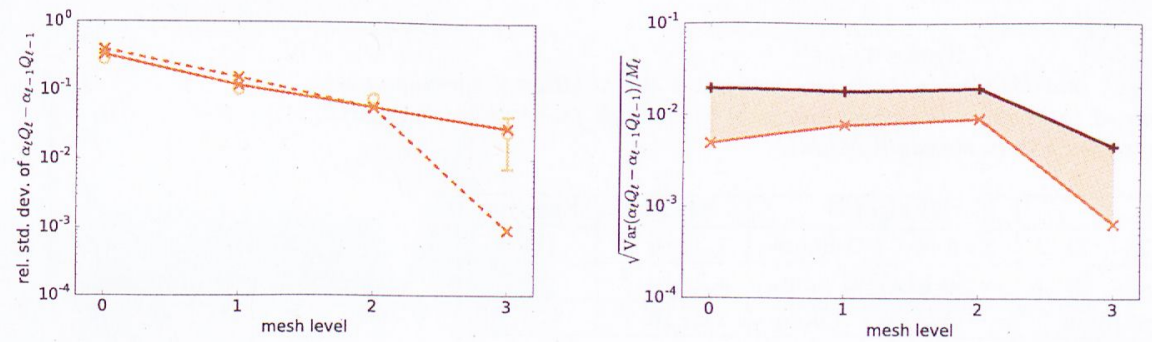

FIG. 6. Estimated variances of differences $\sigma_{\ell \ell-1}^{2}$ (left) required in (33) and the resulting estimated sampling errors $\varepsilon_{\ell}$ (right) defined in (40) on each level. In the left plot, circles depict measurements $\sigma_{\ell, \ell-1}^{2}$ with the associated error bars, the solid line depicts the resulting inferred values, and the dashed line depicts the adjusted values $\tilde{\sigma}_{\ell, \ell-1}^{2}$ from (40), where empirically optimal control variate coefficients are applied. In the right plot, the brown line indicates estimated errors on each level after the initial MLMC warm-up iteration, whereas the orange line and the corresponding region indicate the estimated error improvements after the final MLMC iteration. The final total statistical error is estimated to be approximately $1.3 \cdot 10^{-2}$.

estimated from the variance of the estimator and the number of samples used in the warm-up stage. These measurements are used within a Bayesian inference framework to fit an exponential decay model for $\sigma_{\ell, \ell-1}^{2}$ w.r.t. "difference" levels $\ell=1,2,3$. In particular, Gaussian error model and uniform prior are assumed, resulting in a simple weighted linear least squares regression on measured $\sigma_{\ell, \ell-1}^{2}$. The solid line depicts the resulting inferred values, which are later used in (33), whereas the dashed line depicts the adjusted values $\tilde{\sigma}_{\ell, \ell-1}^{2}$ from (40), where empirically optimal control variate coefficients are applied. The resulting statistical errors at both MLMC iterations (warm-up and final) are decreasing w.r.t. the increasing resolution level. At the final MLMC iteration, the errors are significantly decreased on all levels when compared to the warm-up iteration, resulting in the total statistical error estimate of approximately $1.3 \cdot 10^{-2}$.

For the optimization of sample numbers $M_{\ell}$ on each level, a prescribed budget of 16 million core hours was used and the optimal (up to rounding to integers) number of samples was determined by (42). Estimated number of samples $M_{\ell}$ for the warm- 

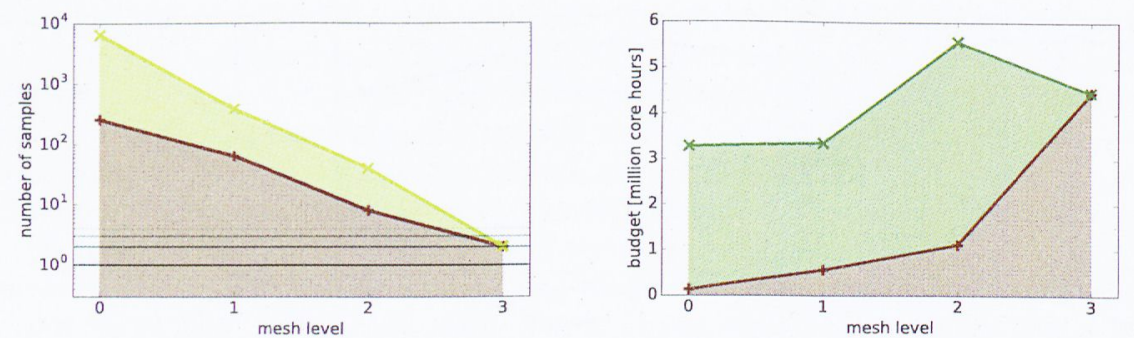

FIG. 7. Estimated number of samples $M_{\ell}$ for the warm-up and final iterations of the $M L C V$ algorithm (left) and the resulting estimated computational budget (right) on each level. Brown lines and the corresponding regions indicate number of samples and computational budget during the initial MLMC warmup iteration, whereas green lines indicate the total accounts of both quantities at the final MLMC iteration; green regions indicating the differences between these two iterations. We observe that despite thousand of samples required on coarser levels of resolution, the required corresponding computational budget is comparable among all levels.

\section{TABLE 1}

Comparison of $M C, M L M C$, and MLCV methods and estimated computational speedups over standard MC. MLCV is estimated to be more than two orders of magnitude faster than plain $M C$, and even more than three times faster than standard $M L M C$.

\begin{tabular}{|c|c|c|c|c|}
\hline & $\left\{M_{\ell}\right\}_{\ell=0, \ldots . L}$ & Budget $\mathcal{B}$ & Error $\varepsilon$ & Speedup \\
\hline MLCV & $6400,384,40,2$ & $16.6 \mathrm{M}$ CPU hours & $1.3 \cdot 10^{-2}$ & 176.8 \\
\hline MLMC & $4352,258,32,3$ & $\sim 50 \mathrm{M}$ CPU hours & $1.3 \cdot 10^{-2}$ & 50.6 \\
\hline MC & $\sim 2$ million & $\sim 2 \mathrm{~B} \mathrm{CPU} \mathrm{hours}$ & $1.3 \cdot 10^{-2}$ & - \\
\hline
\end{tabular}

up and final iterations of the MLCV algorithm are depicted in Figure 7, together with the resulting estimated computational budget on each level. We observe that a significantly larger number of samples is required on the coarser levels of resolution owing to a strong reduction in level difference variances $\tilde{\sigma}_{\ell, \ell-1}^{2}$, which are also highest at the coarsest resolution levels. However, the required computational budget is comparable across all levels; such a multilevel approach achieves a significant (more than two orders of magnitude) reduction in statistical error (i.e., in the variance of the statistical estimators), while at the same time keeping the deterministic error (bias) small, which is controlled by the resolution of the finest level.

In Table 1, a comparison of MC, MLMC, and MLCV methods and estimated computational speedups is provided for a target statistical error of $1.3 \cdot 10^{-2}$. Note, that the number of samples on the same resolution as the finest level in both MLMC and MLCV and the resulting computational budget required for MC simulations are estimated by

$$
M_{\mathrm{MC}}=\left\lceil\frac{\sigma_{L}}{\varepsilon_{\mathrm{MLCV}}}\right\rceil, \quad \mathbb{W}_{\mathrm{MC}}=M_{\mathrm{MC}} \mathbb{W}_{L} .
$$

MLCV is estimated to be more than two orders of magnitude faster than the plain MC method, and even more than three times faster than standard MLMC method without optimized control variate coefficients. The overall computational budget of MLCV was only approximately 8 times larger than a single FVM solve on the finest resolution level. 

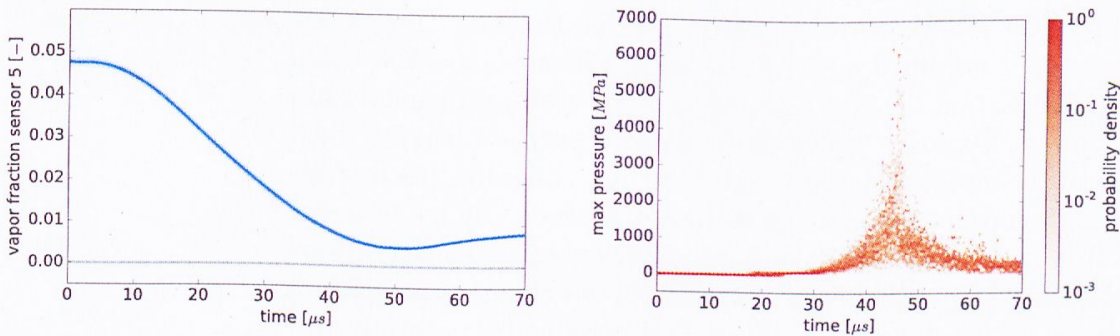

FIG. 8. Uncertainties in the cloud gas volume (mean values with $50 \%$ and $90 \%$ confidence intervals, left) and global maximum pressure (scaled empirical histogram, right) within the cloud during the collapse. Since all initial cloud configurations contain the same number of equally sized cavities, very low uncertainties are observed in the evolution of the total gas volume. However, the statistical spread of the peak pressure is especially wide at its maximum value, implying a large uncertainty in the damage potential of the cavitation collapse.
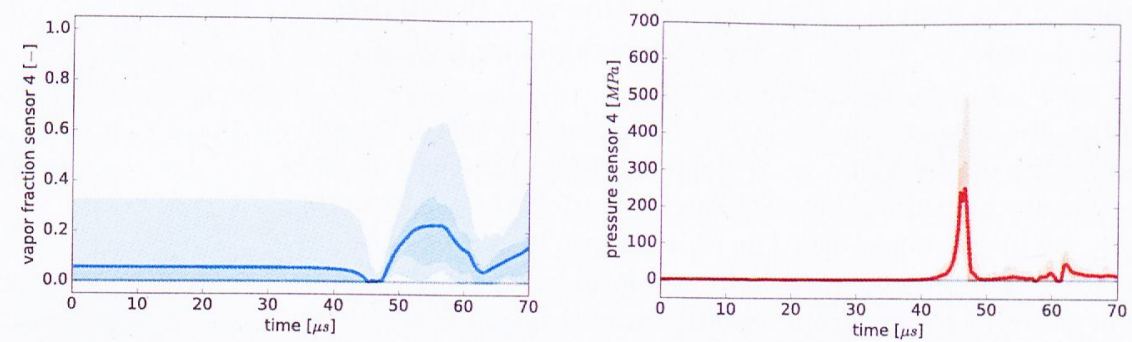

FIG. 9. Uncertainties (mean values with $50 \%$ and $90 \%$ confidence intervals) in the gas volume (left) and pressure (right) sensor at the center of the cloud during the collapse. Notice that the statistical spread of the peak sensor pressure is especially wide at its maximum value and the postcollapse increase in the gas fraction during the rebound stage.
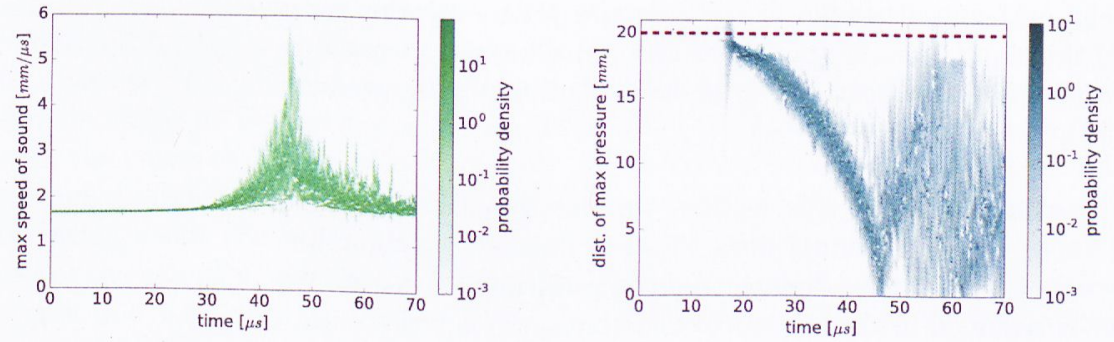

FIG. 10. Uncertainties (probability density functions) in the maximum speed of sound (left) and peak pressure location (right) during the collapse. The dashed line indicates the initial location of the cloud surface.

3.3. Statistics for temporal QOIs. MLMC statistical estimates are depicted in Figures 8, 9, and 10. The statistical spread of the maximum (in physical domain) pressure is especially wide at its peak (in time) value, implying a large uncertainty in the damage potential of the cavitation collapse. To the best of our knowledge, such

Copyright (C) by SIAM. Unauthorized reproduction of this article is prohibited. 
full probability density functions (PDFs) are reported here for the first time when using the MLMC methodology for nonlinear systems of conservations laws. To obtain such estimates, level-dependent kernel density estimators were used, with maximum bandwidth determined using a well-known "solve-the-equation" method $[70,66]$ Such empirical PDFs are significantly more valuable in engineering, compared to less informative mean/median and deviations/percentiles estimators, especially for bimodal probability distributions often encountered in such nonlinear systems of conservations laws due to the presence of shocks [47]. In multilevel statistical quantiles and empirical PDFs, individual estimates on each resolution level are first obtained separately, and then all estimates are combined using a telescoping sum analogous to the definition provided in (32).

In Figure 8, uncertainties in the cloud gas volume (represented by the gas fraction sensor number 5 , located at the center $\mathrm{c}=(50 \mathrm{~mm}, 50 \mathrm{~mm}, 50 \mathrm{~mm})^{\top}$ with $20 \mathrm{~mm}$ radius (hence containing the entire cloud) and global maximum pressure within the cloud are measured during the entire collapse of $70 \mu \mathrm{s}$. As all initial cloud configurations contain the same number of equally sized cavities, very low uncertainties are observed in the evolution of the total cloud gas volume. However, the statistical spread of the peak pressure is especially wide at its maximum value, indicating a strong necessity for UQ in such complex multiphase flows.

In Figure 9, uncertainties are measured in the gas volume fraction sensor $\alpha_{c}$ and pressure sensor $p_{c}$ at the center of the cloud as defined in (44). Similarly as for the observations of peak pressure behavior, the statistical spread of the peak sensor pressure is especially wide at its maximum value. The postcollapse increase in the gas fraction indicates the presence of a rebound stage. During this stage, the postcollapse gas fraction consistently (in terms of confidence intervals) exceeds precollapse values, indicating the presence of induced outgoing rarefaction waves.

In Figure 10, uncertainties in the maximum speed of sound and the peak pressure location distance from the center of the cloud are measured during the entire collapse of $70 \mu \mathrm{s}$. The uncertainty in the maximum speed of sound is a direct consequence of large uncertainties in the global peak pressure. However, on the contrary, the uncertainty in the distance of the peak pressure location from the cloud center is much smaller, i.e., the temporal-spatial profile of the pressure wave evolution as it travels from the surface of the cloud towards the center has a much lower uncertainty (when compared to the large observed uncertainties in the global maximum pressure estimates).

3.4. Statistics for spatial QoIs. In this section, we plot the statistical estimates of QoIs extracted along one-dimensional lines that are cut as a straight line through the center of the cloud in the three-dimensional computational domain. We note that radial symmetry is assumed in the statistical distribution of cavities within the cloud and, hence, such one-dimensional statistical estimates through the center of the cloud are sufficient to represent the global uncertainty in the entire threedimensional domain. The objective of extracted one-dimensional line plots is to provide a better insight into uncertainty structures at the center of the cloud by plotting all statistical estimates in a single plot. The line is cut at a specific physical simulation time, when the peak pressure is observed and, hence, is slightly different for every sample. In order to reduce volatility in global maximum pressure measurements and, hence, the choice of the collapse time, we smoothen the observed peak pressure measurements with a Gaussian kernel of width $0.5 \mathrm{~mm}$ by means of a fast Fourier transform (refer to [71] for methodological details). Statistical estimates for such ex- 

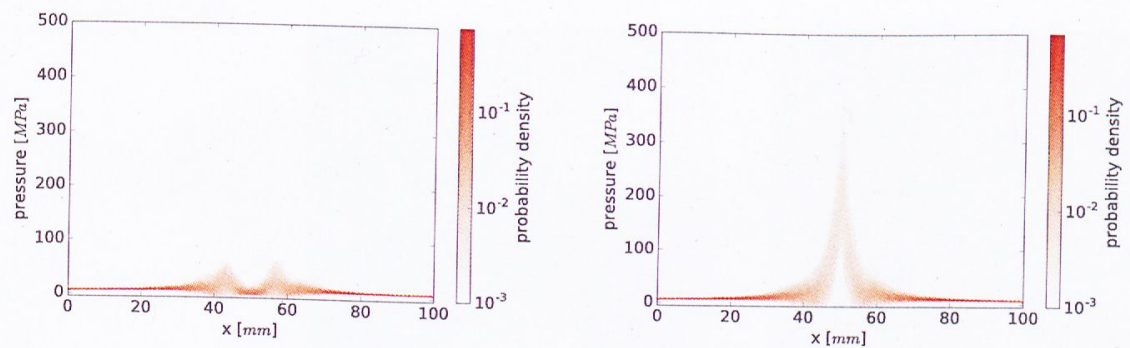

FIG. 11. MLMC-estimated uncertainties (PDFs) of the extracted pressure line at the precollapse time ( $t=43 \mu \mathrm{s}$, left) and at the time of largest peak pressure $(\sim 46 \mu \mathrm{s}$, right). The resulting uncertainty in encountered pressures increases significantly at the final collapse stage, with largest spreads observed near the epicenter of the cloud cavitation collapse.

tracted one-dimensional lines for pressure at different stages of the collapse process are provided in Figure 11.

The uncertainties are estimated using the MLMC methodology in the extracted pressure along the line in the $x$-direction (with coordinates $y=50 \mathrm{~mm}$ and $z=50 \mathrm{~mm}$ fixed) at the precollapse time $t=43 \mu$ s and at the time of largest peak pressure, which occurs approximately at $46 \mu$ s. The time of largest peak pressure depends on the initial cloud configuration and, hence, is a random variable, varying in each statistical realization. We observe that the resulting uncertainty in encountered pressures increases significantly at the final collapse stage, and the largest spreads are observed near the epicenter of the cloud cavitation collapse, where the damage potential is the highest.

3.5. Analysis of linear and nonlinear dependencies. Statistical estimates reported in the previous sections indicate that even though the initial cloud setup is very similar for all realizations, i.e., equal count of cavity bubbles, identical cloud radius, and cavity radii ranges (which resulted in very small uncertainties for the cloud volume reported in Figure 8), and equal initial gas and liquid pressures, the resulting peak pressure uncertainty is very large, as seen in Figures 9 and 11.

Hence, it is only the actual configuration (or distribution) of the cavities inside the cloud that can have such an amplifying (or attenuating) effect on the peak pressures. The main scope of this section is to investigate various QoIs that could potentially explain the cause of such nonlinear effects. The set of selected candidate metrics for the cloud configuration includes skewness (asymmetry) of the initial spatial cavity distribution inside the cloud, cloud interaction parameter $\beta$, and distance (from the center of the cloud) of the central cavity (i.e., the cavity closest to the center). Cloud skewness is a measure of asymmetry of the cloud and is estimated by a statistical centered third moment of the distribution of cavity locations along each of the three spatial axes. All quantities from this set of candidate metrics are tested for linear statistical correlations with the observed values of peak pressure, peak pressure distance from the center of the cloud, peak pressure at the sensor at the center of the domain, and collapse time (when largest peak pressure occurs). In addition, we have also tested for statistical correlations among QoIs themselves, such as peak pressure location and the location of the centermost cavity in the cloud. The results are provided as a Hinton diagram in Figure 12.

We observe multiple significant direct and inverse linear statistical correlations between candidate metrics and QoIs:

Copyright (C) by SIAM. Unauthorized reproduction of this article is prohibited. 

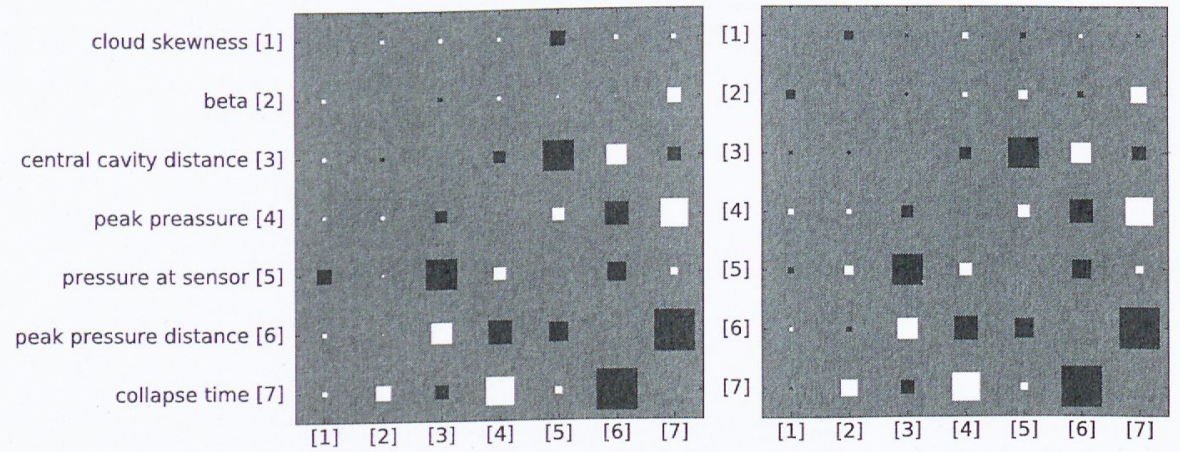

FIG. 12. Hinton diagram indicating pairwise linear statistical correlations between candidate metrics and selected QoIs. White squares indicate positive and black squares indicate negative correlations. The size of the square indicates the magnitude of the correlation in the interval $[0,1]$. Cavities within the entire cloud and only in the core of the cloud are considered in the left and right plots, respectively.

- mild inverse correlation between cloud skewness and pressure sensor readings, mainly a consequence of the central sensor placement within the cloud;

- strong correlation between the location of central cavity and location of peak pressure (w.r.t. cloud center), confirming prior observations in [67] that peak pressures in the cloud are observed within cavities that are near the center of the cloud;

- strong inverse correlations between peak pressure location and peak pressure magnitude, indicating that highest peak pressures are most probable near the center of a cloud;

- moderate correlation between $\beta$ and collapse time, since large $\beta$ values can be a consequence of a large gas fraction in the cloud.

Despite numerous correlations explaining the statistical spread of observed pressures, the influence of cloud interaction parameter $\beta$ remains undetermined. To this end, we consider cloud skewness and $\beta$ only for the core of the cloud. We have identified the core of the cloud to be a region around the center of the cloud where uncertainties in peak pressure are largest, resulting in a spherical core with radius of $10 \mathrm{~mm}$, based on results in Figure 11. In this case, correlations involving respective metrics such as cloud skewness and $\beta$ for the core of the cloud, are observed to be more significant:

- mild direct correlation between $\beta$ and pressure sensor readings, indicating stronger collapse for clouds with higher cloud interaction parameter $\beta$ due to stronger pressure amplification;

- mild inverse correlation between cloud skewness and $\beta$.

Overall, such insight into statistical correlations provides a very informative description of interdependencies between cloud properties and observed collapse pressures, suggesting direct causal relations w.r.t. cloud nonskewness, interaction parameter $\beta$, and proximity of the central cavity to the cloud center. In particular, the polar symmetry of the cloud appears to be a critical component of the collapse dynamics. We did restrain, however, from restricting simulations to artificial "random yet completely symmetric" clouds, rarely observed in real world applications, in order to keep the UQ estimated unbiased with respect to bubble distributions. 

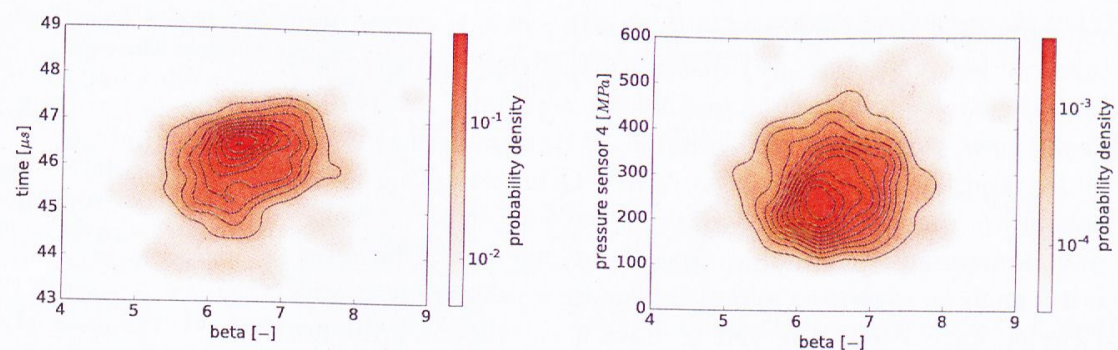

FIG. 13. Joint PDFs of cloud interaction parameter $\beta$ with collapse time (left) and the resulting pressure sensor reading (right). Higher values of cloud interaction parameter $\beta$ are more likely to cause larger and more delayed collapse pressures.
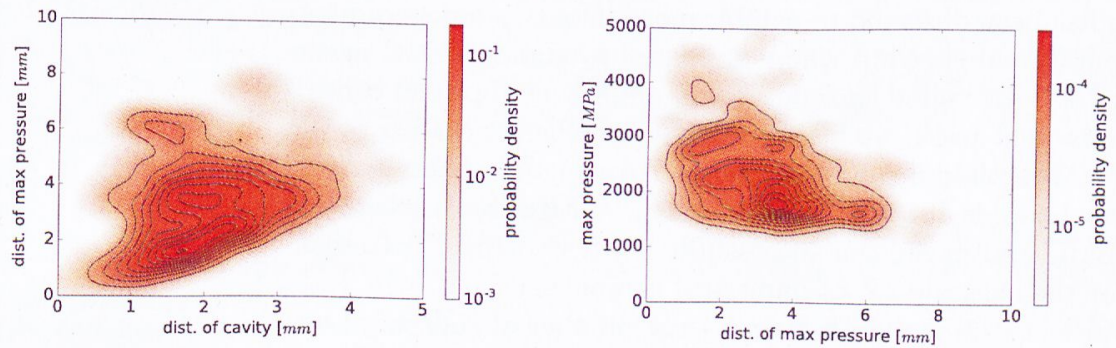

FIG. 14. Joint PDFs of location of central cavity and location of peak pressure (left), and location of peak pressure and peak pressure magnitude (right). The location of the central cavity bubble correlates strongly with peak pressure location, which itself exhibits strong inverse correlations with the magnitude of the collapse pressures, explaining the wide confidence intervals observed in Figures 8 and 11.

Due to the nonlinear nature of the multiphase equations and the collapse process, nonlinear dependencies among candidate metrics and QoIs could be present, which might be inaccurately captured by the estimates of linear statistical correlations in Figure 12. We tested this hypothesis by estimating the full joint probability distribution for the pairs of significantly correlated candidate metrics and pressure behavior observations. In Figures 13 and 14, we provide results for a selected subset of tested correlation pairs, where the strongest and most relevant correlations were observed.

Joint probability distributions are consistent with linear correlation estimates obtained in Figure 12 and, additionally, provide valuable insight into nonlinear dependencies among QoIs. Obtained results provide a good global overview of causal links between cloud structure and collapse pressures and motivate further analysis to determine the complex mechanisms governing the dynamics of such large and complex cloud cavitation phenomena. We would also like to refer to an ongoing extensive (deterministic) parameter study [67] which investigates such causal links for a wider range of cloud sizes, cavity counts, and cloud interaction parameter $\beta$ values.

4. Summary and conclusions. We have presented a nonintrusive multilevel MC methodology for UQ in multiphase cloud cavitation collapse flows, together with novel MLCV coefficients which maintain the efficiency of the algorithm even for weak correlations among resolution levels and deliver significant variance reduction improve-

Copyright (C) by SIAM. Unauthorized reproduction of this article is prohibited. 
ments. We have conducted numerical experiments for cavitating clouds containing 500 cloud cavities, which are randomly (uniformly) distributed within the specified $20 \mathrm{~mm}$ radius, and the radii of the cavity follow a log-normal distribution. The results of these numerical experiments have revealed significant uncertainties in the magnitude of the peak pressure pulse, emphasizing the relevance of UQ in cavitating flows. Furthermore, statistical correlation and joint PDF estimates have revealed potential underlying causes of this phenomenon. In particular, spatial arrangement characteristics of the cloud and its core, such as skewness, cloud interaction parameter $\beta$, and the position of the central cavity have been observed to have a significant influence on the resulting pressure amplification intensities during the collapse process.

All numerical experiments were performed by coupling an open source PyMLMC framework with Cubism-MPCF, a high performance petascale finite volume solver. The evolution of collapsing clouds has been simulated by explicit time stepping subject to a CFL stability constraint on a hierarchy of uniform, structured spatial meshes. Efficient MLMC sampling has been observed to exhibit more than two orders of magnitude in estimated computational speedup when compared to standard MC methods, with an additional factor 3 estimated speedup due to empirically optimal control variate coefficients. In the present work, we have observed the efficient scaling of the proposed hybrid MLCV-FVM method up to the entire MIRA supercomputer consisting of half a million cores. Considering that fault-tolerance mitigation mechanisms are implemented in PyMLMC and have been successfully used, we expect it to scale linearly and be suitable for the exascale era of numerical computing.

The proposed MLCV-FVM can deal with a very large number of sources of uncertainty. In the problems presented here, 2,000 sources of uncertainty are needed to fully describe the random initial configuration of the collapsing cloud. To the best of our knowledge, currently no other methods (particularly deterministic methods such as quasi-MC, stochastic Galerkin, stochastic collocation, PGD, ANOVA, or stochastic FVM) are able to handle this many sources of uncertainty, in particular, for nonlinear problems with solutions which exhibit pathwise low regularity and possibly nonsmooth dependence on random input fields. Furthermore, the proposed methodology is well suited for multilevel extensions of Markov chain MC methods for Bayesian inversion [33, 39].

The present multilevel methodology is a powerful general purpose technique for quantifying uncertainty in complex flows governed by hyperbolic systems of nonlinear conservation laws such as cloud cavitation flow problems.

Acknowledgments. JŠ would like to thank Stephen Wu for his contribution and fruitful discussions on the optimal variance reduction techniques in the multilevel MC estimators, and Timothy J. Barth for hosting him as a scientific visitor at NASA Ames Research Center, Mountain View, CA, and for collaborations on robust kernel density estimators.

\section{REFERENCES}

[1] N. A. Adams AND S. J. SchmidT, Shocks in cavitating flows, in Bubble Dynamics and Shock Waves, C. F. Delale, ed., Shock Wave Sci. Technol. Ref. Libr. 8, Springer, Berlin, 2013 pp. $235-256$

2] M. R. BAFR AND J. W. NunZIATO, A two-phase mixture theory for the deflagration-todetonation transition (DDT) in reactive granular materials, Int. J. Multiphase Flow, 12 (1986), pp. 861-889.

[3] A. BARTH, C SChwaB, AND N. Zollinger, Multi-level MC method for elliptic PDEs with stochastic coefficients, Numer. Math., 119 (2011), pp. 123-161.

Copyright (C) by SIAM. Unauthorized reproduction of this article is prohibited. 
[4] C. Bierig and A. Chernov, Convergence analysis of multilevel Monte Carlo variance estimators and application for random obstacle problems, Numer. Math., 130 (2015), pp. 579-613, https://doi.org/10.1007/s00211-014-0676-3.

[5] J. R. Blake, M. C. Hooțon, P. B. Robinson, and R. P. Tong, Collapsing cavities, toroida bubbles and jet impact, Philos. Trans. R. Soc. Lond. Ser. A Math. Phys. Eng. Sci., 355 (1997), pp. 537-550.

[6] L. Bonfiglio, P. Perdikaris, S. Brizzolara, and G. E. Karniadakis, A Multi-fidelity Framework for Investigating the Performance of Super-cavitating Hydrofoils under Uncertain Flow Conditions, AIAA Non-Deterministic Approaches, AIAA-2017-1328, AIAA, Reston, VA, 2017, https://arc.aiaa.org/doi/abs/10.2514/6.2017-1328.

[7] N. Bremond, M. Arora, C.-D. OHt, And D. Lohse, Controlled multibubble surface cavitation, Phys. Rev. Lett., 96 (2006), 224501.

[8] C. E. Brennen, Cavitation and Bubble Dynamics, Oxford University Press, New York, 1995.

[9] C. E. Brennen, Cavitation in medicine, Interface Focus, 5 (2015), 20150022.

[10] C. E. Brennen, G. Reisman, And Y.-C. Wang, Shock waves in cloud cavitation, Twenty-First Symposium on Naval Hydrodynamics, National Academy Press, Washington, DC, 1997, pp. 756-771.

[11] E. A. Brujan, T. Ikeda, And Y. Matsumoto, Shock wave emission from a cloud of bubbles, Soft Matter, 8 (2012), pp. 5777-5783.

[12] F. CAPpello, Fault tolerance in petascale/exascale systems: Current knowledge, challenges and research opportunities, Int. J. High Perform. Comput. Appl., 23 (2009), pp. 212-226.

[13] G. L. Chahine, Pressures generated by a bubble cloud collapse, Chem. Eng. Commun., 28 (1984), pp. 355-367.

[14] Q. Y. Chen, D. Gottlieb, And J. S. Hesthaven, Uncertainty analysis for steady flow in a dual throat nozzle, J. Comput. Phys., 204 (2005), pp. 378-398.

[15] K. A. Cliffe, M. B. Giles, R. Scheichl, and A. L. Teckentrup, Multilevel Monte Carlo methods and applications to elliptic PDEs with random coefficients, Comput. Vis. Sci., 14 (2011), pp. 3-15.

[16] N. Collier, A.-L. Haji-Ali, F. Nobile, E. von Schwerin, And R. Tempone, A continuation multilevel Monte Carlo algorithm, BIT, 55 (2015), pp. 399-432.

[17] P. M. Congedo, E. Goncalves, And M. G. Rodio, About the uncertainty quantification of turbulence and cavitation models in cavitating flows simulations, Eur. J. Mech. B Fluids, 53 (2015), pp. 190-204.

[18] C. C. Coussios And R. A. Roy, Applications of acoustics and cavitation to noninvasive therapy and drug delivery, Annu. Rev. Fluid Mech., 40 (2008), pp. 395-420.

[19] D. P. Schmidt, S. J. Schmidt, M. Minatsch, M. Thalhamer, And N. A. Adams, Assessment of the prediction capabilities of a homogeneous cavitation model for the collapse characteristics of a vapour-bubble cloud, in WIMRC 3rd International Cavitation Forum, Coventry, UK, 2011.

[20] T. J. Dodwell, C. Ketelsen, R. Scheichl, And A. L. Teckentrup, A hierarchical multilevel Markov chain Monte Carlo algorithm with applications to uncertainty quantification in subsurface flow, SIAM/ASA J. Uncertain. Quantif., 3 (2015), pp. 1075-1108, https://doi. org/10.1137/130915005.

[21] D. Elsakout, M. Christie, And G. Lord, Multilevel Markov chain Monte Carlo (MLM$C M C$ ) for uncertainty Bayesian framework for uncertainty quantification, in SPE North Africa Technical Conference and Exhibition 2015, Technical report SPE-175870-MS, SPE, Richardson, TX, 2015

[22] H. Fairbanks, A. Doostan, C. Ketelsen, and G. Iaccarino, A low-rank control variate for multilevel Monte Carlo simulation of high-dimensional uncertain systems, J. Comput. Phys., 341 (2016), pp. 121-139. https://doi.org/10.1016/j.jcp.2017.03.060.

[23] M. B. Giles, Multilevel Monte Carlo methods, Acta Numer., 24 (2015), pp. 259 328, https: //doi.org/10.1017/S096249291500001X.

[24] M. B. Giles, Multi-level Monte Carlo path simulation, Oper. Res., 56 (2008), pp. 607-617.

[25] M. B. Giles, Multilevel Monte Carlo path simulation, Oper. Res., 56 (2008), pp. 607-617, https://doi.org/10.1287/opre.1070.0496.

[26] M. B. Giles AND C. Reisinger, Stochastic Finite Differences and Multilevel Monte Carlo for a Class of SPDEs in Finance, preprint, Oxford-Man Institute of Quantitative Finance and Mathematical Institute, University of Oxford, Oxford, 2011.

[27] D. GotTlieB AND D. XIU, Galerkin method for wave equations with uncertain coefficients, Commun. Comput. Phys., 3 (2008), pp. 505-518.

[28] S. Gottlieb And C.-W. Shu, Total variation diminishing Runge-Kutta schemes, Math. Comp., 67 (1998), pp. $73-85$.

Copyright (C) by SIAM. Unauthorized reproduction of this article is prohibited. 
[29] P. E. Hadidoukas, P. Angelikopoulos, C. Papadimitriou, and P. Koumoutsakos, $114 U$ : $A$ high performance computing framework for Bayesian uncertainty quantification of complex models, J. Comput. Phys., 284 (2015), pp. 1-21.

[30] P. E. Hadjidoukas, D. Rossinelli, B. Hejazialhosseini, and P. Koumoutsakos, From 11 to 14.4 PFLOPS: Performance optimization for finite volume flow solver, in Proceedings of the 3rd International Conference on Exascale Applications and Software, University of Edinburgh, Edinburgh, 2015.

[31] P. E. Hadjidoukas, D. Rossinelli, F. Wermelinger, J. Sukys, U. Rasthofer, C. Conti, B. Hejazialhosseini, And P. Koumoutsakos, High throughput simulations of two-phase flows on Blue Gene/Q, in Parallel Computing: On the Road to Exascale, Proceedings of the International Conference on Parallel Computing, ParCo 2015, Edinburgh, Scotland, Adv. Parallel Comput. 27, IOS Press, Amsterdam, 2015, pp. 767-776.

[32] S. Heinrich, Multilevel Monte Carlo methods, in Large-scale Scientific Computing, Third International Conference 2001, Sozopol, Bulgaria, 2001, Lecture Notes in Comput. Sci. 2179, Springer, Berlin, 2001, pp. 58-67.

[33] V. H. HoAng. C. SCHwab, ANd A. M. Stuart, Sparse MCMC GPC finite element methods for Bayesian inverse problems, ETHZ Research Collection, Report 2012-23, 23, 2012, https: //doi.org/10.3929/ethz-a-010395062.

[34] X. Y. Hu, B. C. Khoo, N. A. Adams, And F. L. Huang, A conservative interface method for compressible flows, J. Comput. Phys., 219 (2006), pp. 553-578.

[35] G.-S. JiAnG AND C.-W. SHu, Efficient implementation of weighted ENO schemes, J. Comput. Phys., 126 (1996), pp. 202-228

[36] E. JOHNSEN AND T. COLONIUS, Implementation of WENO schemes in compressible multicomponent flow problems, J. Comput. Phys., 219 (2006), pp. 715-732

[37] E. JOHNSEn AND T. Colonius, Numerical simulations of non-spherical bubble collapse, J. Fluid Mech., 629 (2009), pp. 231-262.

[38] A. K. Kapila. R. Menikoff, J. B. Bdzil, S. F. Son, And D. S. Stewart, Two-phase modeling of deflagration-to-detonation transition in granular materials: Reduced equations, Phys. Fluids, 13 (2001), pp. 3002-3024.

[39] C. Ketelsen, R. Scheichl, And A. L. Teckentrup, A hierarchical multilevel Markov chain Monte Carlo algorithm with applications to uncertainty quantification in subsurface flow, SIAM/ASA J. Uncertain. Quantif., 3 (2015), pp. 1075-1108.

40] E. Lauer, X. Y. Hu, S. Hickel, And N. A. AdAms, Numerical investigation of collapsing cavity arrays, Phys. Fluids, 24 (2012), 052104

[41] S. LI, Tiny bubbles challenge giant turbines: Three gorges puzzle, Interface Focus, 5 (2015), 20150020 .

[42] G. Lin, C. H. SU, AND G. E. Karniadakis, The stochastic piston problem, Proc. Natl. Acad. Sci. USA, 101 (2004), pp. 15840-15845.

43] K. Lye, Multilevel Monte-Carlo for Measure Valued Solutions, Technical report 2016-51, Seminar for Applied Mathematics, ETH, Zürich, Switzerland, 2016, https://www.sam.math. ethz.ch/sam_reports/reports_final/reports2016/2016-51.pdf.

[44] X. MA AND N. ZABARAS, An adaptive hierarchical sparse grid collocation algorithm for the solution of stochastic differential equations, J. Comput. Phys., 228 (2009), pp. 3084-3113.

[45] R. Mentkoff and B. J. Plohr, The Riemann problem for fluid flow of real materials, Rev. Modern Phys, 61 (1989), pp. 75-130.

[46] S. Mishra And C. Schwab, Sparse tensor multi-level Monte Carlo finite volume methods for hyperbolic conservation laws with random initial data, Math. Comp., 280 (2012), pp. $1979-$ 2018.

[47] S. Mishra, Ch. Schwab, And J. Šukys, Multi-level Monte Carlo finite volume methods for nonlinear systems of conservation laws in multi-dimensions, J. Comput. Phys., 231 (2012), pp. 3365-3388.

[48] S. Mishra. Ch. Schwab, And J. Sukys, Multilevel Monte Carlo finite volume methods for shallow water equations with uncertain topography in multi-dimensions, SIAM J. Sci. Comput. 34 (2012), pp. B761-B784.

[49] S. Mishra, C. SCHWAB, AND J. ŠUkYs, Multi-level Monte Carlo finite volume methods for uncertainty quantification of acoustic wave propagation in random heterogeneous layered medium, J. Comput. Phys., 312 (2016), pp. 192-217, https://doi.org/10.1016/j.jcp. 2016. 02.014.

[50] K. A. Mørch, On the collapse of cavity clusters in flow cavitation, in Cavitation and Inhomogeneities in Underwater Acoustics, W. Lauterborn, ed., Springer, Berlin, 1980, pp. 95-100.

[51] K. A. MørCH, Energy considerations on the collapse of cavity clusters, Appl. Sci. Res., 38 (1982), pp. 313-321

Copyright (C) by SIAM. Unauthorized reproduction of this article is prohibited. 
[52] F. MüLLER, Stochastic Methods for Uncertainty Quantification in Subsurface flow and Transport Problems, Dissertation, ETH Zurich, Zurich, 2014.

[53] F. Müller, P. Jenny, And D. W. Meyer, Multilevel Monte Carlo for two phase flow and Buckley-Leverett transport in random heterogeneous porous media, J. Comput. Phys., 250 (2013), pp. 685-702, https://doi.org/10.1016/j.jcp.2013.03.023.

[54] A. Murrone and H. Guillard, A five equation reduced model for compressible two phase flow problems, J. Comput. Phys., 202 (2005), pp. 664-698.

[55] F. Nobile And F. Tesei, A Multi Level Monte Carlo method with control variate for elliptic PDEs with log-normal coefficients, Stoch. Partial Differ. Equ. Anal. Comput., 3 (2015), pp. 398-444, https://doi.org/10.1007/s40072-015-0055-9.

[56] L. Parussini, D. Venturi, P. Perdikaris, and G. E. Karniadakis, Multi-fidelity Gaussian process regression for prediction of random fields, J. Comput. Phys., 336 (2017), pp. 36-50, https://doi.org/10.1016/j.jcp.2017.01.047.

[57] S. PAuLI, Optimal Number of Multilevel Monte Carlo Levels and Their Fault Tolerant Application, Dissertation, ETH Zurich, Zurich, 2014.

[58] S. Pauli, M. Kohler, And P. Arbenz, A fault tolerant implementation of multi-level Monte Carlo methods, in Parallel Computing - Accelerating Computational Science and Engineering (CSE), Adv. Parallel Comput. 23, IOS Press, Amsterdam, 2014, pp. 471-480.

[59] B. Peherstorfer, K. Willcox, And M. Gunzburger, Optimal Model Management for Multifidelity Monte Carlo Estimation, SIAM J. Sci. Comput., 38 (2016), pp. A3163-A3194.

[60] B. Peherstorfer, K. Willcox, and M. Gunzburger, Optimal model management for multifidelity Monte Carlo estimation, SIAM J. Sci. Comput., 38 (2016), pp. A3163-A3194, https://doi.org/10.1137/15M1046472.

[61] B. Peherstorfer, K. Willcox, And M. Gunzburger, Survey of Multifidelity Methods in Uncertainty Propagation, Inference, and Optimization, SIAM Rev., 60 (2018), pp. 550591.

[62] G. Perigaud and R. Saurel, A compressible flow model with capillary effects, J. Comput. Phys., 209 (2005), pp. 139-178.

[63] G. Poette, B. Després, AND D. LuCor, Uncertainty quantification for systems of conservation laws, J. Comput. Phys., 228 (2009), pp. 2443-2467.

[64] T. J. POINSOT AND S. K. LeLE, Boundary conditions for direct simulations of compressible viscous flows, J. Comput. Phys., 101 (1992), pp. 104-129.

[65] M. Raissi, P. PERdikaris, and G. E. Karniadakis, Inferring solutions of differential equations using noisy multi-fidelity data, J. Comput. Phys., 335 (2017), pp. 736-746, https://doi.org/ 10.1016/j.jcp.2017.01.060.

[66] U. Rasthofer, F. Wermelinger, P. Hadijdoukas, and P. Koumoutsakos, Large scale simulation of cloud cavitation collapse, Procedia Comput. Sci., 108 (2017), pp. 1763-1772, https://doi.org/10.1016/j.procs.2017.05.158.

[67] U. Rasthofer, F. Wermelinger, J. Sukys, P. Hadijdoukas, and P. Koumoutsakos, Parameter Study in Cloud Cavitation Collapse, manuscript.

[68] D. Rossinelli, B. Hejazialhosseini, P. E. Hadjidoukas, C. Bekas, A. Curioni, A. Bertsch, S. Futral, S. J. Schmidt, N. A. Adams, And P. Koumoutsakos, $11 \mathrm{PFLOP/s} \mathrm{simula-}$ tions of cloud cavitation collapse, in Proceedings of the International Conference on High Performance Computing, Networking, Storage and Analysis, SC '13, New York, 2013, ACM, New York.

[69] D. P. SCHMidt AND M. L. Corradini, The internal flow of diesel fuel injector nozzles: A review, Internat. J. Engine Res., 2 (2001), pp. 1-22.

[70] S. J. Sheather And M. C. Jones, A reliable data-based bandwidth selection method for kernel density estimation, J. R. Stat. Soc.. Ser. B Stat. Methodol., 53 (1991), pp. 683-690.

[71] J. S. Simonoff, Smoothing Methods in Statistics, Springer Ser. Statist., Springer, New York, 1996, https://doi.org/10.1007/978-1-4612-4026-6.

[72] J. ŠUKYs, Adaptive load balancing for massively parallel multi-level Monte Carlo solvers, in PPAM 2013, Part I, Lecture Notes in Comput. Sci. 8384, Springer, Berlin, 2014, pp. 47-56.

[73] J. SukYs, Robust Multi-level Monte Carlo Finite Volume Methods for Systems of Hyperbolic Conservation Laws with Random Input Data, Dissertation, ETH Zurich, Zurich, 2014.

[74] J. Šukys, PyMLMC, https://github.com/cselab/PyMLMC (2017).

[75] J. Šukys, S. Mishra, And C. Schwab, Static load balancing for multi-level Monte Carlo finite volume solvers, PPAM 2011, Part I, Lecture Notes in Comput. Sci. 7203, Springer, Heidelberg, 2012, pp. 245-254.

[76] A. Tiwari, J. B. Freund, And C. Pantano, A diffuse interface model with immiscibility preservation, J. Comput. Phys., 252 (2013), pp. 290-309.

Copyright (C) by SIAM. Unauthorized reproduction of this article is prohibited. 
[77] A. Tiwari, C. PAntano, And J. B. Freund, Growth-and-collapse dynamics of small bubble clusters near a wall, J. Fluid Mech., 775 (2015), pp. 1-23.

[78] E. F. Toro. M. Spruce, And W. Speares, Restoration of the contact surface in the HLLRiemann solver, Shock Waves, 4 (1994), pp. 25-34.

[79] J. Tryoen, O. L. Maitre, M. Ndjinga, And A. Ern, Intrusive projection methods with upwinding for uncertain non-linear hyperbolic systems, J. Comput. Phys., 229 (2010), pp. 6485-6511.

[80] X. WAN AND G. E. KARNIADAKIS, Long-term behaviour of polynomial chaos in stochastic flow simulations, Comput. Methods Appl. Mech. Engrg., 195 (2006), pp. 5582-5596.

[81] J. Williamson, Low-storage Runge-Kutta schemes, J. Comput. Phys., 35 (1980), pp. 48-56.

[82] J. A. S. Witteveen, A. Loeven, And H. BiJl, An adaptive stochastic finite element approach based on Newton-Cotes quadrature in simplex elements, Comput. \& Fluids, 38 (2009), pp. $1270-1288$.

[83] D. XIU AND J. S. HESTHAVEN, High-order collocation methods for differential equations with random inputs, SIAM001X J. Sci. Comput., 27 (2005), pp. 1118-1139.

[84] K. YAmamoto, Investigation of bubble clouds in a cavitating jet, in Mathematical Fluid Dynamics, Present and Future, Springer, Tokyo, 2016, pp. 349-373.

Copyright (C) by SIAM. Unauthorized reproduction of this article is prohibited. 